\title{
Council meeting
}

\section{September 2018}

13:30 to $16: 00$ approx.

Council Room 1, 25 Canada Square, London E14 5LQ

Public business

1. Attendance and introductory remarks

Nigel Clarke

2. Declarations of interest

Public items

All

3. Minutes of last meeting

Public session on 12 July 2018

Nigel Clarke

4. Workshop summary - 12 July 2018

Nigel Clarke

5. Actions and matters arising

Nigel Clarke

6. Performance monitoring and annual plan progress report

18.09.C.01

For noting

Duncan Rudkin

7. Registration assessment - June $\mathbf{2 0 1 8}$

18.09.C.02

For noting

Damian Day

8. Pharmacist independent prescribing

18.09.C.03

For noting

Priya Warner

9. Standards for the education and training of pharmacist independent

18.09.C.04

prescribers: consultation report

Damian Day

For noting

10. Scoping research on mechanisms in fitness to practise processes to

18.09.C.05

eliminate discrimination

Claire Bryce-Smith

For noting

11. Council member appointments 2019

18.09.C.06 For approval

Laura McClintock

12. Minutes from the Audit and Risk committee - 17 July 2018

18.09.C.07

Digby Emson

13. Any other public business

Nigel Clarke 


\section{Confidential business}

14. Declarations of interest

All

Confidential items

15. Minutes of the last meeting

Nigel Clarke

Confidential session on 12 July 2018

16. Confidential actions and matters arising

Nigel Clarke

17. Confidential minutes from the Audit and Risk committee - 17 July

18.09.C.08 2018

Digby Emson

18. Strategic risk register

18.09.C.09

For discussion

Pascal Barras

19. Any other confidential business

Nigel Clarke

Date of next meeting

Thursday, 11 October 2018 


\section{General}

Pharmaceutical

Council

Minutes of the Council meeting held on Thursday 12 July 2018 at 25 Canada

Square, London at 13:30

TO BE CONFIRMED 13 SEPTEMBER 2018

Minutes of the public session

Present

Nigel Clarke (Chair)

Evelyn McPhail

Digby Emson

Arun Midha

Mark Hammond

Berwyn Owen

Mohammed Hussain

David Prince

Jo Kember

Samantha Quaye

Alan Kershaw

Jayne Salt

Elizabeth Mailey

Apologies

Mary Elford

Megan Forbes

In attendance

Duncan Rudkin (Chief Executive and Registrar)

Claire Bryce-Smith (Director of Insight, Intelligence and Inspection)

Matthew Hayday (Interim Director of Fitness to Practise)

Francesca Okosi (Director of People)

Mark Voce (Interim Director of Education and Standards)

Laura McClintock (Chief of Staff)

Rachael Oliver (Head of Communications)

Priya Warner (Head of Policy and Standards)

Damian Day (Head of Education)

Osama Ammar (Head of Revalidation) 
Helen Dalrymple (Council Secretary)

\section{Attendance and introductory remarks}

31.1. The Chair welcomed all present to the meeting. Apologies had been received from Mary Elford and Megan Forbes.

\section{Declarations of interest}

32.1. Council agreed that members would make any declarations of interest before each item.

33. Minutes of the last meeting

33.1. The minutes of the public session held on the $\mathbf{7}$ June 2018 were confirmed as a fair and accurate record.

34. Workshop summary - 7 June 2018

34.1. The Chair noted that some items covered in workshop could be described as 'work in progress'. The next summary would endeavour to capture the threads of discussion around these.

34.2. Council noted the discussions from the workshop.

\section{Actions and matters arising}

35.1. Action ref. 31.6. from the meeting on 6 July 2017 said that a report would be brought to Council on equality, diversity and inclusion in fitness to practise processes. Duncan Rudkin (DR) told members that a supplier had been appointed to work with the organisation. Scoping was under way and had highlighted the complexity of the matter. There were a variety of options of how to take the report forward. A paper would be brought to the Council meeting in September that would summarise the work so far and ask Council for a steer on next steps.

35.2. All other actions were in hand or due to be covered at this meeting. 


\section{Report of the Gosport Independent Panel; PSA Lessons Learned Review; and, the Professor Sir Norman Williams Review of Gross Negligence Manslaughter in Healthcare.}

36.1. Laura McClintock (LM) presented 18.07.C.01. This paper outlined a number of recent developments in the external context and updated Council on the organisation's response.

36.2. Members said that the issue of how whistle-blowers and those raising concerns were treated had struck them throughout the reports and that this was an area that could benefit from discussion with other regulators on how to improve it. In particular, the reports emphasised the need for a culture shift, to ensure that regulators were treating families and those raising concerns with respect and supporting them through the fitness to practise process.

36.3. Members also discussed the issue of the quality and consistency of expert witnesses, as highlighted in the Williams Review. LM highlighted that expert witnesses were not used often in the GPhC's fitness to practise process. However, it was assumed that this was likely to change over time as pharmacy professionals worked more clinically. This meant that the issues may become relevant in a way that they hadn't historically. It was suggested that expert witnesses possessed a certain set of skills that did not just relate to their knowledge. Perhaps in the future more could be done to identify and develop those who could carry out this role. These issues would be considered through the action plan going forward.

36.4. Members also discussed the joint emerging concerns protocol, developed under the governance of the Health and Social Care Regulators Forum. The grading tool used in the protocol was discussed. One member felt that it could potentially contribute to a negative view of complainants that was unhelpful and that on occasion those who had complained multiple times did have valid concerns and should not be disregarded. LM explained that the grading system within the protocol was simply an informal tool, to support consistency and help organisations to make decisions about sharing information. It did not override statutory or other requirements, decisions would still be made on a case by case basis, and were not determined by grading.

36.5. It was also suggested that it would be helpful to update the design of the protocol summary diagram to prevent the assumption that all routes led to no further action in cases where the protocol was not initiated.

36.6. Council pointed out that these reports were all in England and that organisations in Scotland and Wales should also be engaged with on these matters. LM said that the regulators that made up the initial group of those that had signed up to the emerging concerns protocol were keen to do this and were inviting those from organisations across the UK to take part. 
36.7. A query was raised about the section in the emerging concerns protocol where the GPhC said that they would like to be informed of concerns about registered pharmacies but later in the same section, referred to a 'pharmacy setting'. Member questioned whether this was accurate. LM said that in part it was a drafting point but also that the first part of the section was being very clear on the GPhC's jurisdiction, which did not extend to all 'pharmacy settings' as such. She would seek to clarify this with the authors.

36.8. Council suggested that the Health and Social Care Advisory Service (HASCAS) report investigating the care and treatment provided on Tawel Fan Ward in North Wales could be added to this paper. Frontline staff needed to be enabled and empowered to identify and report systemic failings.

36.9. DR told Council that changing culture to encourage this was linked to the standards for registered pharmacies, which placed a clear responsibility on pharmacy owners to create an environment in which their professional staff could meet their professional standards. Throughout other regulators as well as within the GPhC, the culture should be one of adhering to standards that are expected of others.

36.10. Members looked forward to an update in the autumn following the publication of the formal Government report and recommendations arising from the Gosport report.

36.11. Council:

i. Noted the response to a number of recent independent inquiries and reports within the healthcare sector; and

ii. Noted the progress update on the joint work of the professional regulators and others to develop a new emerging concerns protocol.

37. Strategic approach to engagement with patients and the public and their elected representatives.

37.1. Rachael Oliver (RO) presented 18.07.C.02, this paper set out the organisation's strategic approach to engaging with patients and the public and their elected representatives.

37.2. There was an update to the paper at para 3.8.; a meeting would take place with Jeane Freeman, the new Cabinet Secretary for Health in Scotland.

37.3. Members asked about engagement with the All-Party Parliamentary Group on Pharmacy (APPG). RO confirmed that this was regular and Council members would be encouraged to attend. The group's report on pharmacy and long-term conditions had recently been published and this would be circulated following the meeting.

ACTION: RO

37.4. It was suggested that the organisation should also engage with the Scottish Health Council and the Integrated Joint Boards in Scotland as they had elected councillors as 
members. RO welcomed the suggestion and said that further suggestions from Council would be most welcome.

37.5. There had been issues with capacity and interest but moving forward developing further links with other organisations would very much be a focus of the Communications team. It was anticipated that the publication of inspection reports would provide a good opportunity to do this.

37.6. Members suggested developing links with some cities where NHS scrutiny bodies had links with local government.

37.7. Council asked for more frequent briefings to enable them to better represent the GPhC in their spheres, RO agreed that this could be provided if members would find it helpful.

37.8. Council members in Scotland and Wales asked to be notified when meetings that they could attend were arranged. RO said that the Directors for Wales and Scotland were very well networked and would welcome Council member involvement where appropriate.

\subsection{Council:}

i. Noted the GPhC's current approach to engaging with patients and the public and their elected representatives; and

ii. Considered plans to further develop their approach going forward.

\section{Rebalancing Medicines Legislation \& Pharmacy Regulation: Consultation}

38.1. Priya Warner (PW) presented 18.07.C.03, which updated Council on the Government consultation 'Rebalancing medicines legislation and pharmacy regulation'

38.2. Members pointed out that the proposals would involve a lot of work for the organisation and hoped that enough resource would be available. PW agreed saying that following a period of analysis this was inevitable. The organisation would strive to be as efficient and effective as possible whilst developing the suite of new standards, some of which would align to existing strands of work.

38.3. Council asked that care was taken with language. At para 3.2. the intention was that changes would increase reporting, learning and improvement rather that it being a fact.

38.4. Concern was expressed over what steps could be taken to protect the superintendent pharmacist in a corporate structure. It was agreed that this would be highlighted in the response to the consultation and that it tied in with the GPhC's guidance for pharmacy owners.

38.5. Council noted the update on the Government consultation and the organisation's approach to and participation in the consultation. 


\section{Learning from the Registration Assessment 2010-2018}

39.1. Damian Day (DD) presented 18.07.C.04. This paper provided Council with a summary of developments in the Registration Assessment 2010-18, summary findings of candidate performance and suggested actions based on the findings.

39.2. Digby Emson declared an interest as the chair of a pharmacy training body and Samantha Quaye declared an interest as she worked for an education provider for pharmacists and pharmacy technicians.

39.3. Members were assured to know that work was ongoing into understanding candidate performance by characteristics. It was important to be careful and measured in identifying what may affect performance and how many factors contributed to candidates' performance, including not only their undergraduate experience but also their pre-registration training and their own capabilities.

39.4. Council wanted to know if the data in this report would be used to trigger earlier accreditation procedures for schools that had higher numbers of poor performers. DD replied that they proposed to allow each school to speak for themselves rather than apply a metric across them all.

39.5. Members suggested that given the long-standing nature of the subject perhaps it would be wise to bring in an external group to look at the data, validate the work that had been done so far and demonstrate how seriously the organisation was taking this matter. DD replied that this was already under consideration.

39.6. Information on the performance of candidates according to what sector they were from would be welcomed; DD said that Health Education England (HEE) were addressing this in England as they commissioned pre-registration training courses.

39.7. Council also asked when there would be some information on any link between entrylevel qualifications and registration assessment pass rates. DD said that this would be available soon - data on entry grades was not available until two years ago, in a couple of months there would be enough data to interrogate.

39.8. Mark Voce (MV) told members that more information could be provided on the 'Assuring Quality in Education' workstream and would circulated after the meeting.

\section{ACTION: MV}

39.9. Members asked whether the use of Oriel had improved the quality of recruitment onto pre-registration. DD explained that they were working closely with HEE and that evaluation was built in to monitor this. They were keen to be as equitable and fair as possible. 
39.10. Council said that the report was most welcome and that it was important to adhere to their role as the regulator and not over-extend their remit. In some cases there was a gap between pre-registration tutors and learning sites which it would be helpful to understand.

39.11. The focus should remain on the whole journey of students up until the registration assessment. Previous reports had shown the benefits and significant impact of role models for students.

39.12. The Council provided feedback on the next steps that were identified in the paper.

\section{Monitoring and governance of revalidation for pharmacy professionals}

40.1. Osama Ammar (OA) presented 18.07.C.05. This paper asked Council to agree an approach to monitoring and governing revalidation for pharmacy professionals following its implementation.

40.2. Members asked how evaluation of revalidation would be conducted. OA replied that it would be carried out in two stages. Over the first two years surveys of registrants' perceptions of the process would be carried out. Once the new system of revalidation had bedded in, evaluation would also be made of the impact that it had on the quality of the records that were being submitted.

40.3. Council would be able to monitor the effectiveness of the advisory group through the performance monitoring report, where another section on revalidation submission rates would be added as well as the other evaluation material, to all of which they would have access.

40.4. The Chair formally recorded Council's thanks to Lord Kirkwood, who had served as chair of the advisory group, for his considerable assistance over a long period of time.

40.5. Council:

i. Approved revisions to the governance structure for revalidation including the disestablishment of the Council assurance group for revalidation

ii. Approved new terms of reference for the advisory group (at appendix one)

iii. Thanked Lord Kirkwood for his work in support of revalidation throughout the process of its development

\section{Gender pay gap reporting 2017}

41.1. Francesca Okosi (FO) presented 18.07.C.06. This paper set out the organisation's gender pay gap (GPG), explained the statutory reporting arrangements and the steps being taken 
to close the GPG. An earlier version of this paper was discussed at the Remuneration Committee meeting on 18 April 2018.

41.2. Council expressed their thanks at the paper being tabled at this meeting and heard that this would be published annually. They were encouraged that there was not a huge divergence in pay across gender.

41.3. Members encouraged the organisation to consider establishing a women's network to add to those described at para 6.5.

41.4. Members asked how the figures would change if the Associates' pay were included in the data. FO replied that it would have some impact. Rates of pay were the same across this group, but how Associates were deployed did cause some difference in pay across gender. The chair of the Appointments Committee was aware of this and work was under way to address it.

41.5. Council noted the $\mathbf{2} 017$ gender pay gap report.

42. Council member appointments 2019

42.1. LM presented 18.07.C.07. This paper asked Council to consider recommendations on the process for filling Council member vacancies arising in March 2019. The vacancies would need to be filled through an open competition process as none of the members were eligible for reappointment.

42.2. In the 'Desirable criteria' section of appendix one, Council discussed D1. Many felt that as there was a lot of change in the pharmacy sector, they would also want to encourage candidates with experience of leadership in the sector of community pharmacy.

42.3. D2 should be updated with 'registrant member' in brackets. It was agreed that it would be very desirable to have at least one member of Council who was a pharmacy technician.

42.4. Members suggested updating D4 to include reference to 'experience of representing the views of patients or carers' as an alternative to direct experience as a patient or carer.

42.5. Council were asked to consider how to encourage a broad and diverse range of suitable candidates, any suggestions would be welcome and should be directed to the Chair.

42.6. Council:

i. Agreed that the five Council member vacancies arising in March 2019 would be filled using an open competition process;

ii. Agreed the updated selection criteria and competencies for new Council member appointments at appendix 1; and

iii. Noted the next steps in the appointments process and timetable. 


\section{Any other business}

43.1. Council expressed their thanks to Claire Bryce-Smith for her work in the introduction of a clinical leadership fellow to the GPhC.

43.2. There being no further public business to discuss the meeting closed at 15:15

Date of the next meeting:

Thursday 13 September 2018 


\section{Meeting paper}

Council on Thursday, 13 September 2018

\section{Public business}

\section{Council Workshop Summary}

\section{Purpose}

To provide an outline note of the discussions at the July Council workshop

\section{Recommendations}

The Council is asked to note the discussions from the workshop

\section{Introduction}

1.1. The Council holds a workshop session alongside its regular Council meetings each month (there are no meetings in January and August). The workshops give Council members the opportunity to:

- $\quad$ interact with and gain insights from staff responsible for delivering regulatory functions and projects;

- $\quad$ receive information on projects during the development stages;

- $\quad$ provide guidance on the direction of travel for work streams via feedback from group work or plenary discussion; and

- $\quad$ receive training and other updates.

1.2. Following each workshop there is a summary of the discussions that took place, presented at the subsequent meeting. This makes the development process of our work streams more visible to the GPhC's stakeholders. Some confidential items may not be reported on in full.

1.3. In the workshop sessions the Council does not make decisions. The sessions are informal discussions to aid the development of the Council's views. 


\title{
2. Summary of the July workshop
}

\subsection{Investments and risk appetite}

Members had a presentation on the various types of assets in which monies could be invested, along with their relative risks.

Following this session, the risk appetite of Council could be assessed and fed into the development of an investment strategy for the organisation.

\subsection{Initial findings from the pharmacist independent prescriber consultation}

Emerging feedback on the main points raised following the end of the consultation on education and training standards for pharmacist independent prescribers was presented to Council. Members discussed this and gave their initial views.

This would assist with the analysis of the consultation responses, which is provided at the meeting of Council in September.

\subsection{Continuing our development of a ten-year vision}

Members had an update on the feedback that they had provided at the last workshop in the item on strategy. There was some discussion on what insight themes could be tracked over the short, medium and longer term. Council were asked to explore the longer-term ambition for their role with regards to patients and the public.

The discussion is informing ongoing strategic planning work, which will return to Council iteratively over the coming months.

\section{Recommendations}

Council is asked to note the discussions from the workshop

\author{
Duncan Rudkin, Chief Executive and Registrar \\ General Pharmaceutical Council \\ duncan.rudkin@pharmacyregulation.org
}

Tel 02037138011

5 September 2018 


\section{Council actions log}

\begin{tabular}{|c|c|c|c|c|c|c|}
\hline Meeting date & Ref. & Action & Owner & $\begin{array}{l}\text { Due } \\
\text { date }\end{array}$ & Status & Comments/update \\
\hline 6 Jul 17 & 31.6. & $\begin{array}{l}\text { Consultation on revised } \\
\text { threshold criteria: A report on } \\
\text { equality, diversity and inclusion in } \\
\text { Fitness to Practise processes } \\
\text { would be brought to Council in } \\
\text { due course. }\end{array}$ & $\begin{array}{l}\text { Claire } \\
\text { Bryce- } \\
\text { Smith }\end{array}$ & Sep 18 & Open & This on the agenda for today's meeting \\
\hline 7 Jun 18 & 25.16 . & $\begin{array}{l}\text { PMR and annual plan progress } \\
\text { report - Education: Members } \\
\text { asked for more information on } \\
\text { how independent prescribing } \\
\text { programmes varied and how } \\
\text { competence was assessed. }\end{array}$ & Mark Voce & Sep 18 & Open & This is covered in today's agenda \\
\hline \multirow[t]{2}{*}{12 Jul 18} & 37.3. & $\begin{array}{l}\text { Strategic approach to } \\
\text { engagement with patients and } \\
\text { the public and their elected } \\
\text { representatives: The APPG's } \\
\text { report on pharmacy and long- } \\
\text { term conditions would be } \\
\text { circulated to members }\end{array}$ & $\begin{array}{l}\text { Rachael } \\
\text { Oliver }\end{array}$ & Sep 18 & Closed & $\begin{array}{l}\text { This was circulated to members on the } 17 \\
\text { July }\end{array}$ \\
\hline & 39.8. & $\begin{array}{l}\text { Learning from the Registration } \\
\text { Assessment 2010-2018: More } \\
\text { information would be circulated } \\
\text { to members on the 'Assuring } \\
\text { Quality in Education' workstream }\end{array}$ & Mark Voce & Sep 18 & Open & $\begin{array}{l}\text { A verbal update will be given at the } \\
\text { meeting }\end{array}$ \\
\hline
\end{tabular}


General

Pharmaceutical

Council

\section{Meeting paper}

Council on Thursday, 13 September 2018

\section{Public business}

\section{Performance Monitoring and Annual Plan Progress Report}

\section{Purpose}

To report to Council on operational and financial performance and progress against the annual plan from April to June 2018.

\section{Recommendations}

The Council is asked to note and comment on:

i. the performance information provided at appendix 1 ; and

ii. the report on progress against the 2018/19 annual plan at appendix 2 .

\section{Introduction}

1.1. This paper reports on operational and financial performance and progress against the annual plan, as part of our business report.

1.2. This report provides an overview of Quarter 1 covering April to June 2018.

1.3. The section below provides an executive summary of key areas to note.

2. Performance Monitoring Report

2.1. Appendix 1 reports on the operational and financial performance of the organisation. It is the first progress report to Council this year.

2.2. Some key areas to note in the reporting this quarter include: 
2.2.1. the impact on the Customer Contact Centre with the release of online functionality and the annual peak of enquiries from students sitting the registration assessment

2.2.2. the continuing increase in fitness to practise concerns received and the focus on our oldest cases

2.2.3. Slight reduction in the number of routine inspections over this period, whilst the number of pharmacies not inspected for 36 months or more decreased for the fifth quarter in succession, representing sustained positive direction of travel.

2.2.4. Standard 2.1, which relates to the adequacy of staffing, has appeared in the top 5 for the second time and was highlighted in the previous quarter as an emerging area of concern

2.2.5. The stability rate has increased since the last quarter

2.3. To provide some further narrative around the above:

2.3.1. It should be noted that new registration activity is generally light over this quarter as there are no key reasons for entry such as immediately following an assessment or at the end of the academic timetable (for pharmacy technicians). However, this quarter has seen continued support for registrants signing up to the new My GPhC account for revalidation, as well as the introduction of online pharmacist registration and the annual peak enquiries from students sitting the registration assessment which has had an impact on our performance reporting.

2.3.2. The Customer Contact Centre has answered almost 15,000 calls in this quarter, equivalent to the busiest months in previous years. Almost 10,000 emails have been answered with the number actioned each month being the three highest totals per month ever recorded. Despite the fact that registrants have had to wait longer to speak to a member of the team, the feedback from the overwhelming majority of registrants has been positive due to the quality of the advice and assistance provided.

2.3.3. The number of concerns received has again increased during this quarter. The average number of concerns per month is now 230. In comparison to Q1 2017/18 a year ago, the total number of cases triaged has increased by $47 \%$. Our total number of cases being worked on at any one time has understandably also increased but only by $20.5 \%$ which demonstrates an increase in productivity.

2.3.4. The number of routine inspections over the period decreased from 1068 to 961 . This is due to several factors including increased planned annual leave due to seasonal holiday periods during this quarter, mandatory training courses, the delivery of local presentations for the registered pharmacies consultation and attendance at the annual joint Conference with the CQC. The number of months since the previous inspection has continued to reduce for those over 48 months and over. Standard 2.1 which relates to the adequacy of staffing has appeared in the top 5 for the second time which is an emerging area of concern but represents around $3 \%$ of pharmacies inspected. 
2.3.5. With regard to headcount, the stability rate (based on the number of permanent employees with more than 12 months employment at the $\mathrm{GPhC}$ ) has increased positively from the previous quarter (January to March 2018) from $81 \%$ to $85 \%$.

2.3.6. The year to date position for the organisation overall is a variance of $£ 262 \mathrm{~K}$ against the revised budget including interest and tax. Work continues on the development of a future financial strategy. Regular updates continue to be provided to the Efficiency and Effectiveness Assurance and Advisory Group (EEAAG) and Council.

\section{Annual plan progress report}

3.1. Appendix 2 reports on progress against the Annual Plan 2018/19. This is the first progress report to Council this year. Whilst activities may have progressed since quarter 1 , reporting remains focused on this period as part of good governance and so that this aligns with reporting mechanisms and timescales elsewhere.

3.2. Although in some cases worded slightly different most of the strategic priorities in $2018 / 19$ are a continuation of those set out in the 2017/18 annual plan. This approach reflects an emerging intent to move away from short-term year-to-year planning, instead taking a much longer-term view of our priorities, planning and resources.

3.3. The content of this report reflects the next stage of planning around our strategic priorities and outlines progress against them.

3.4. The strategic priorities we have carried over in our reporting from last year include:

- Building on our data, information, intelligence and insight capability (formally worded as 'Developing our data and insight strategy')

- Securing assurance and promoting improvement in registered pharmacies (formally worded as 'Developing our approach to regulating registered pharmacies to provide assurance and encourage improvement')

- Improving standards of care through regulation of education and training (formally worded as 'Setting the standards and quality assuring the initial education and training for pharmacists and pharmacy technicians')

- Transforming our organisation, our services and processes (formally worded 'Transforming our services and the way we work')

3.5. Developing our approach to fitness to practise is a new strategic priority for $2018 / 19$.

3.6. The 'providing further assurance to the public that pharmacy professionals are meeting the standards' (revalidation) work has been incorporated into the 'improving standards of care through regulation of education and training' strategic priority. 
3.7. The work scoped out last year under 'promoting professionalism through the standards for pharmacy professionals and related guidance' has concluded. Nevertheless, work continues on the following (i) safe and effective pharmacy team guidance, (ii) forthcoming guidance on prescribing, as well as (iii) preparation of work with regard to rebalancing and the Department of Health and Social Care consultation which will require us to draft standards for chief pharmacists, superintendent pharmacists and responsible pharmacists.

3.8. Status of work in this quarter is as follows:

\begin{tabular}{|l|l|l|}
\hline Strategic Priorities & Status & $\begin{array}{l}\text { Direction } \\
\text { of travel }\end{array}$ \\
\hline Building our data, information, intelligence and insight capability & $\mathrm{G}$ & \\
\hline Developing our approach to fitness to practise & $\mathrm{A}$ & $\begin{array}{l}\text { New } \\
\text { Strategic } \\
\text { Priority }\end{array}$ \\
\hline $\begin{array}{l}\text { Securing assurance and promoting improvement in registered } \\
\text { pharmacies }\end{array}$ & $\mathrm{G}$ & \\
\hline $\begin{array}{l}\text { Improving standards of care through regulation of education and } \\
\text { training }\end{array}$ & $\mathrm{A}$ & \\
\hline Transforming our organisation, our services and processes & A & \\
\hline
\end{tabular}

3.9. Two items remain amber from the previous quarter, these are

- Improving standards of care through regulation of education and training - this work programme is extensive, highly complex and now also includes the revalidation and unregistered pharmacy team work. We have also accelerated the timescale for consulting on the revised initial education and training standards for pharmacists meaning there are some complex issues to resolve fully in a slightly shorter timeframe.

It should be noted that the revalidation work whilst now incorporated into this workstream was reported as green in the last quarter.

- Transforming our organisation, our services and processes - this remains amber although there has been good delivery against actions. Following approval of the revised budget for the 2018-19 financial year, activities continue to be reviewed and prioritised. 
Developing our approach to fitness to practise is a new strategic priority so has not previously been reported against. The activities in quarter 1 have been delivered on time but the planned work could be subject to delay due to the resources available to initiate and deliver these projects.

Two items remain as green. These are 'securing assurance and promoting improvement in registered pharmacies' and 'building on our data, information, intelligence and insight capability'.

3.10. We acknowledge that the 'transforming our organisation, our services and processes' section is a mixture of activities with various pieces of work being progressed under this heading. Both the internal auditors and the Efficiency and Effectiveness Assurance and Advisory Group (EEAAG) have recommended a top down review of the projects and programmes that meet the defined purpose of transformation. EEAAG will continue to oversee the transition from transformation to something else as part of our new emerging strategy with updates provided to Council, when appropriate.

3.11. With regards to reporting, we've looked to provide transparency on how we have progressed against the timetable we set ourselves for the annual plan year; where we have proceeded in accordance with the timetable; those areas where we have fallen behind or where we might be ahead of where we thought we would be. Further explanation with regards to the timetable is provided in the commentary.

3.12. Council will note that in previous progress reports we stated that to challenge ourselves in our planning, we need to progressively introduce specific success measures going forwards. We accept that progress in this area was limited last year, both from a planning and governance perspective. Inclusion of success measures for the annual plan this year represents progress in this area and an indication of intent moving forwards. However, we recognise that this is not just about management process but a wider change in culture as well, with everyone knowing what success looks like.

\section{Equality and diversity implications}

4.1. Our aim is to embed equality, diversity and inclusion in both our role as a regulator and an employer. We will continue to look at how we can monitor and demonstrate our progress towards this aim. 


\section{Communications}

5.1. The development and publication of this report is reflective of our commitment to openness and transparency concerning our performance. We continue to carry out specific communications on each of the areas of reported performance. This includes information on our website, wider communications through the media and directly through our own publications and communications materials. These activities are designed to reach all our key interest groups including patients and their representatives, pharmacy professionals and their employees, education providers and others.

5.2. Internal communications on our annual plan including the detail that sits underneath will be important as we go through a period of change. There will need to be transparent and specific communications around key stages of the plan in order to inform and engage with staff.

\section{Resource implications}

6.1. Resource implications are addressed within the report.

6.2. The allocation of resources required to progress with the annual plan as well as normal operational delivery remains a key consideration in developing our budget and fee setting proposals.

\section{Risk implications}

7.1. The strategic risk register will continue to be reviewed as part of our management framework and risks will be recorded and reviewed in relation to our work.

7.2. Main risks associated with the delivery of the annual plan are included as part of the annual plan progress report.

7.3. With regards to operational performance, failure to maintain an accurate register and/or carry out our other regulatory functions efficiently and effectively could have implications on patient safety, and a significant impact on the GPhC's reputation.

7.4. Failure to accurately forecast/budget for revenues and expenditure could lead to inappropriate or inconsistent fee policies which could have an adverse impact on the GPhC's reputation. 


\section{Monitoring and review}

8.1. Council will receive a performance monitoring and annual plan progress report on a quarterly basis, providing an update on the delivery of the GPhC's regulatory functions and progress against the annual plan

8.2. We continue to be mindful of and look to feed in learning from planning and reporting previously as part of our commitment to continuous learning and improvement.

\section{Recommendations}

The Council is asked to note and comment on:

i. the performance information provided at appendix 1; and

ii. the report on progress against the annual plan at appendix 2

\section{Duncan Rudkin, Chief Executive \\ General Pharmaceutical Council \\ duncan.rudkin@pharmacyregulation.org}

Tel 02037137805 
General

Pharmaceutical

Council

\section{Performance Monitoring Report: end June 2018}


1. Customer services

1.1 Registrations

\begin{tabular}{|c|c|c|c|c|c|c|}
\hline & \multirow{2}{*}{ Route to Register } & \multicolumn{4}{|c|}{ 2017/18 } & \multirow{2}{*}{$\begin{array}{c}2018 / 19 \\
\text { Q1 }\end{array}$} \\
\hline & & Q1 & Q2 & Q3 & Q4 & \\
\hline \multirow{5}{*}{ Pharmacists } & Total & 94 & 2,321 & 597 & 243 & 86 \\
\hline & UK & 60 & 2,260 & 561 & 200 & 56 \\
\hline & EEA & 30 & 20 & 22 & 31 & 24 \\
\hline & Non-EU/EEA & 4 & 41 & 14 & 12 & 4 \\
\hline & Need clarification & & & & & 1 \\
\hline \multirow{4}{*}{ Pharmacy technicians } & Total & 194 & 350 & 441 & 211 & 207 \\
\hline & UK & 191 & 343 & 438 & 209 & 207 \\
\hline & EEA & 3 & 5 & 2 & 2 & \\
\hline & Non-EU/EEA & & 2 & 1 & & \\
\hline Registered pharmacies & & 127 & 102 & 79 & 82 & 73 \\
\hline
\end{tabular}

Includes new joiners and restorations up to $30^{\text {th }}$ June 2018

New registration activity is generally light over this quarter as there are no key reasons for entry such as immediately following an assessment or the end of the academic timetable (for pharmacy technicians). 


\subsection{Registration Totals}

\begin{tabular}{|l|c|c|c|}
\hline & Total & Budgeted & Variance \\
\hline Pharmacists & 55,256 & 55,162 & +94 \\
\hline Pharmacy technicians & 23,391 & 23,403 & -12 \\
\hline Registered pharmacies & 14,330 & 14,344 & -14 \\
\hline
\end{tabular}

Register totals as at 30 th June 2018

1.3

\section{Median application processing times for pharmacists}

\begin{tabular}{|l|c|l|c|}
\hline \multicolumn{2}{|c|}{$\begin{array}{c}\text { Median application processing times for } \\
\text { pharmacists (working days) }\end{array}$} & \multicolumn{2}{c|}{$\begin{array}{c}\text { Median application processing times for } \\
\text { pharmacy technicians (working days) }\end{array}$} \\
\hline Application receipt to approval & 2.5 & Application receipt to approval & 10 \\
\hline Application receipt to entry & 1 & Application receipt to entry & 9 \\
\hline
\end{tabular}

Medians calculated for applications during the period $1^{\text {st }}$ April to $30^{\text {th }}$ June 2018 


\subsection{Contact Centre}

\begin{tabular}{|c|c|c|c|c|c|c|c|c|}
\hline \multirow{2}{*}{ Phone } & \multicolumn{3}{|c|}{ 2016/17 } & \multicolumn{4}{|c|}{$2017 / 18$} & \multirow{2}{*}{$\frac{2018 / 19}{Q 1}$} \\
\hline & Q2 & Q3 & Q4 & Q1 & Q2 & Q3 & Q4 & \\
\hline Calls made to GPhC & 18,539 & 13,081 & 9,176 & 14,024 & 17,131 & 11,968 & 8,596 & 24,005 \\
\hline $\begin{array}{l}\text { Calls answered within } 20 \text { seconds (KPI } \\
>80 \%)\end{array}$ & $73.6 \%$ & $60.0 \%$ & $62.6 \%$ & $49.0 \%$ & $67.5 \%$ & $71.3 \%$ & $89.4 \%$ & $23.5 \%$ \\
\hline Calls abandoned (KPI < 5\%) & $4.0 \%$ & $9.8 \%$ & $9.0 \%$ & $11.8 \%$ & $5.8 \%$ & $5.9 \%$ & $1.8 \%$ & $38.0 \%$ \\
\hline \multicolumn{9}{|l|}{ Correspondence } \\
\hline $\begin{array}{l}\text { Emails actioned within } 2 \text { days (KPI > } \\
90 \%)\end{array}$ & $92.6 \%$ & $80.0 \%$ & $89.3 \%$ & $98.6 \%$ & $97.3 \%$ & $99.6 \%$ & $99.5 \%$ & $51.9 \%$ \\
\hline
\end{tabular}

The performance figures for this quarter relate to the paper published in the June Council paper, highlighting the implementation of revalidation and the impact on the Customer Contact Centre (CCC). That paper highlighted the overall success of the implementation and the risk mitigation that had been put in place while noting that we underestimated demand in two key areas: the volume of registrants signing up to the new MyGPhC in the first few months and the volume of calls where registrants need to be guided through the sign-up process. This quarter has also seen the introduction of online pharmacist registration and the annual peak enquiries from students sitting the registration assessment.

The CCC has answered almost 15,000 calls in this quarter, equivalent to the busiest months in previous years. Almost 10,000 emails have been answered with the number actioned each month being the three highest totals per month ever recorded. Despite the fact that registrants have had to wait longer to speak to a member of the team, the feedback from the overwhelming majority of registrants has been positive due to the quality of the advice and 
assistance provided. This reflects the briefing and training provided to the CCC prior to the launch of revalidation and the decisions we have taken to guide registrants through the sign-up process which has extended the length of individual calls.

We continue to update information on our website with frequently asked questions and are continuing our communication activity to raise awareness of revalidation and promote the resources available on our website and those of professional bodies. Automated messages on the telephone lines are informing registrants that this is an exceptionally busy period to help manage expectations. We continue to use resources flexibly to answer calls and emails, including using appropriately trained members from other teams. The CCC is now fully staffed and the senior customer service representative is in post enabling the CCC manager to focus more time on planning and use of resources. 


\subsection{Continuing Professional Development}

There will be no further reporting for the old model for CPD.

New reporting will commence after the renewal deadline of $31^{\text {st }}$ October 2018 (December Council). 


\section{Fitness to Practise (FtP)}

\subsection{Fitness to Practise performance standards ${ }^{1}$}

\begin{tabular}{|l|c|c|c|c|c|c|}
\hline \multicolumn{2}{|c}{} & \multicolumn{2}{c|}{$\mathbf{2}$} & \multicolumn{2}{c|}{$\mathbf{2 0 1 8 / 1 9}$} \\
\cline { 3 - 8 } & & Q1 & Q2 & Q3 & Q4 & Q1 \\
\hline All cases triaged during this period & No. & 462 & 563 & 611 & 667 & 681 \\
\hline \multirow{2}{*}{ Of which cases triaged within 3 working days } & No. & 458 & 540 & 381 & 485 & 438 \\
\cline { 2 - 8 } & $\%$ & $99.1 \%$ & $95.9 \%$ & $62.4 \%$ & $72.7 \%$ & $62.8 \%$ \\
\hline \multirow{2}{*}{ Of which cases triaged within 5 working days } & No & & & 532 & 601 & 576 \\
\cline { 2 - 8 } & $\%$ & & & $87.1 \%$ & $90.1 \%$ & $84.6 \%$ \\
\hline
\end{tabular}

Cases closed 1 April 2018 to 30 June 2018, which may have been opened at any time.

\begin{tabular}{|c|c|c|c|c|c|c|}
\hline & & \multicolumn{4}{|c|}{$2017 / 18$} & \multirow{2}{*}{$\frac{2018 / 19}{Q 1}$} \\
\hline & & Q1 & Q2 & Q3 & Q4 & \\
\hline All stream 1 cases closed pre-IC & No. & 148 & 153 & 212 & 223 & 249 \\
\hline \multirow{2}{*}{ Of which closed within 3 months } & No. & 138 & 127 & 177 & 193 & 209 \\
\hline & $\%$ & $93.2 \%$ & $83.0 \%$ & $83.9 \%$ & $86.5 \%$ & $83.9 \%$ \\
\hline $\begin{array}{l}\text { All stream } 2 \text { cases closed pre-IC or referred to } \\
\text { the IC[1] }\end{array}$ & No. & 157 & 123 & 179 & 192 & 160 \\
\hline \multirow{2}{*}{ Of which closed or referred within 10 months } & No. & 131 & 106 & 148 & 144 & 120 \\
\hline & $\%$ & $83.4 \%$ & $86.2 \%$ & $82.7 \%$ & $73.0 \%$ & $75.0 \%$ \\
\hline All cases closed or referred at IC & No. & 53 & 36 & 18 & 45 & 28 \\
\hline \multirow{2}{*}{ Of which reach IC within 12 months } & No. & 18 & 16 & 13 & 29 & 13 \\
\hline & $\%$ & $34.0 \%$ & $44.4 \%$ & $72.2 \%$ & $64.4 \%$ & $46.4 \%$ \\
\hline
\end{tabular}

${ }^{1}$ Minor update made to a few figures in previous quarters due to end of year reconciliation 


\begin{tabular}{|c|c|c|c|c|c|}
\hline No. & 18 & 18 & 14 & 17 & 7 \\
\hline$\%$ & $64.3 \%$ & $62.0 \%$ & $77.7 \%$ & $70.8 \%$ & $41.2 \%$ \\
\hline
\end{tabular}

The number of concerns received has again increased during this quarter. The average number of concerns received per month is now 230 . In comparison to Q1 2017/18, the total number of cases triaged has increased by $47 \%$. Our total number of cases being worked on at any one time has understandably also increased but only by $20.5 \%$ which demonstrates an increase in productivity. Following a real push at the end of the 2017/18 financial year, it was anticipated that quarter 1 would be quieter in terms of productivity. The closure figures for both stream 1 and stream 2 cases however has remained positive despite the increase in concerns.

There has been a drop in the number of cases closed within 24 months. This is as a result of a focus on our oldest cases which can be seen when comparing the age profile data below. During quarter 1 there has been a concerted effort to dispose of the oldest cases as well as keep the newer cases moving through the process in a timely manner. Our aim is to reduce cases over 12 months significantly by the end of this financial year and we can address this through added resource. There are a number of vacancies within the directorate which need to be filled to ensure we can manage the increasing concerns whilst maintaining throughput and reducing the older case load. Recruitment is currently underway and we hope to have these posts filled by Q3.

\subsection{Caseload age profile}

\begin{tabular}{|c|c|c|c|c|c|c|}
\hline \multirow{2}{*}{\multicolumn{2}{|c|}{ Age profile }} & \multicolumn{4}{|c|}{$2017 / 18$} & \multirow{2}{*}{$\begin{array}{c}2018 / 19 \\
Q 1\end{array}$} \\
\hline & & Q1 & Q2 & Q3 & Q4 & \\
\hline Under 6 months & $\%$ & $57.4 \%$ & $58.3 \%$ & $58.8 \%$ & $60.8 \%$ & $60.3 \%$ \\
\hline 6-12 months & No. & 130 & 177 & 157 & 153 & 152 \\
\hline \multirow{2}{*}{ 12-14 months } & No. & 32 & 24 & 30 & 22 & 39 \\
\hline & $\%$ & $4.9 \%$ & $3.1 \%$ & $4.0 \%$ & $2.9 \%$ & $5.1 \%$ \\
\hline \multirow{2}{*}{15 months old and over } & No. & 116 & 119 & 123 & 121 & 110 \\
\hline & $\%$ & $17.8 \%$ & $15.5 \%$ & $16.3 \%$ & $16.0 \%$ & $19.3 \%$ \\
\hline
\end{tabular}




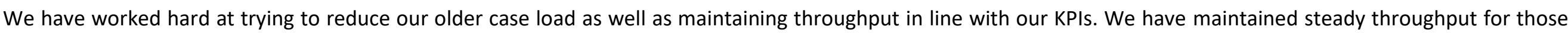

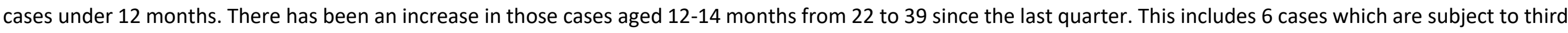
party investigations and are on hold.

\subsection{Cases over 15 months}

\begin{tabular}{|c|c|c|c|c|c|c|}
\hline \multirow{2}{*}{\multicolumn{2}{|c|}{ Age profile }} & \multicolumn{4}{|c|}{$2017 / 18$} & \multirow{2}{*}{$\begin{array}{c}2018 / 19 \\
\text { Q1 } \\
40 \\
\end{array}$} \\
\hline & & $\frac{\text { Q1 }}{46}$ & $\frac{\text { Q2 }}{48}$ & $\frac{\text { Q3 }}{67}$ & $\begin{array}{l}\text { Q4 } \\
65\end{array}$ & \\
\hline 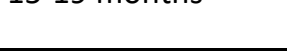 & $\%$ & $39.7 \%$ & $40.3 \%$ & $54.5 \%$ & $53.7 \%$ & $36.4 \%$ \\
\hline 20-24 months & No. & 30 & 29 & 17 & 20 & 34 \\
\hline \multirow{2}{*}{ 25-29 months } & No. & 23 & 17 & 20 & 20 & 11 \\
\hline & $\%$ & $19.8 \%$ & $14.3 \%$ & $16.3 \%$ & $16.5 \%$ & $10.0 \%$ \\
\hline \multirow{2}{*}{ 30-34 months } & No. & 6 & 11 & 10 & 6 & 14 \\
\hline & $\%$ & $5.2 \%$ & $9.2 \%$ & $8.1 \%$ & $5.0 \%$ & $12.7 \%$ \\
\hline $40-42$ months & $\%$ & $0.9 \%$ & $1.6 \%$ & $1.6 \%$ & $2.5 \%$ & $2.7 \%$ \\
\hline \multirow{2}{*}{ 43-49 months } & No. & 4 & 6 & 2 & 1 & 3 \\
\hline & $\%$ & $3.4 \%$ & $5.1 \%$ & $1.6 \%$ & $0.8 \%$ & $2.7 \%$ \\
\hline \multirow{2}{*}{50 months or more } & No. & 1 & 0 & 1 & 1 & 1 \\
\hline & $\%$ & $0.9 \%$ & $0.0 \%$ & $0.8 \%$ & $0.8 \%$ & $1.0 \%$ \\
\hline
\end{tabular}

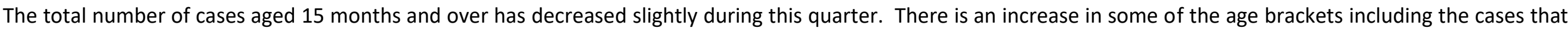

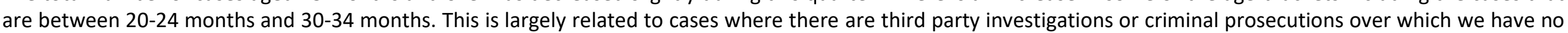


influence or control. The total number of cases which are over 12 months and remain on hold due to third party investigations during quarter 1 is 38 , just over $25 \%$ of the total case load over 12 months.

Following closer scrutiny of all cases aged 12 months and over together with the increase in resources due to come, we anticipate a decrease in these figures during quarter 2 and quarter 3.

One open case has been opened for more than 50 months. The principal hearing before the Fitness to Practise Committee was listed twice in 2017. The first hearing was postponed because of an application for disclosure from the registrant, which was detailed very close to the date of the hearing. The second hearing was adjourned partway through because the chair of the panel considering the case resigned. The Chair of the Fitness to Practise Committee determined that the hearing needed to start again from the beginning. The Committee started the case in quarter four, however, it could not complete its consideration in the time given. Case management was undertaken during quarter four. The case was considered in July and was closed within quarter two of 2018/19. The table above will reflect this in the next reporting period. 


\subsection{Cases closed by stage ${ }^{2}$}

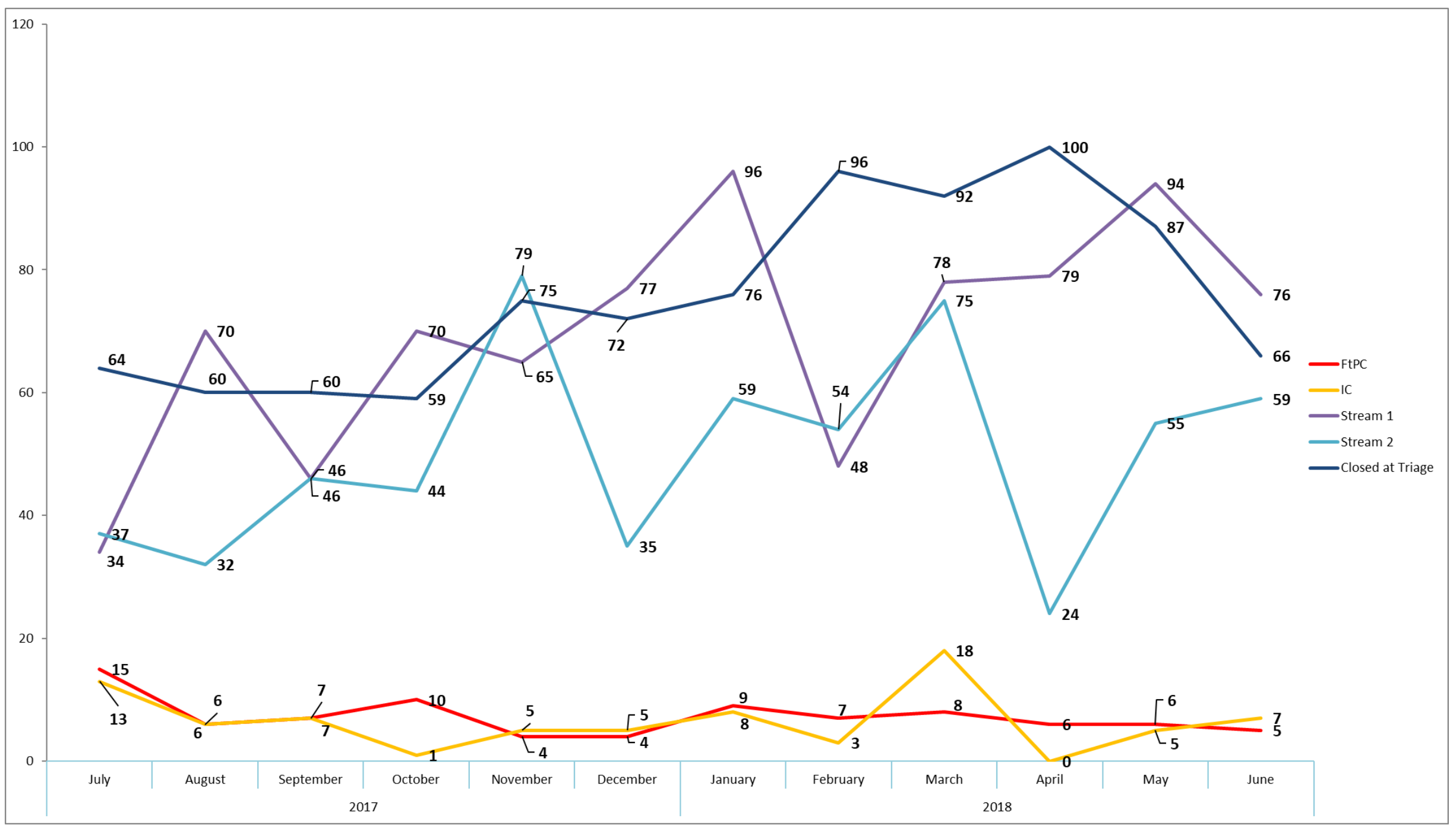

${ }^{2}$ The graph shows closures only. This excludes cases referred to IC as they are not deemed to be closed for the purposes of this graph. 


\subsection{DBS referrals}

The GPhC's Disclosure and Barring Service (DBS) and Disclosure Scotland (DS) Referrals Panel met twice and considered 5 matters during this quarter, of which 3 were referred to DBS (covering England and Wales) and none referred to DS.

\subsection{Appeals}

No appeals were brought in this quarter.

\subsection{Interim Orders}

The Fitness to Practise Committee considered 9 applications for interim orders during this quarter. It agreed a postponement for three applications allowing a further 7 days in two cases and 14 days in one case. One case was postponed allowing the registrant time to access legal representation, one case was postponed due to the registrant being out of the country and wanting to attend, the third case was postponed to allow for further medical evidence to be obtained. Six interim suspension orders were imposed (five for 18 months and one for 12 months). One order for interim conditions was imposed for 12 months and two applications were refused with no order imposed.

During this quarter, the median period for the time taken from receipt of information identifying the need for a potential order and the application being heard increased to 3.4 weeks. In part, this was due to the postponement of two cases in quarter 4 of 2017/18 which increased the time taken as well as three postponements during this quarter as detailed above. 


\section{Inspection}

\subsection{Inspections undertaken}

\begin{tabular}{|l|c|c|c|}
\hline & $\begin{array}{c}\text { Routine } \\
\text { inspections }\end{array}$ & $\begin{array}{c}\text { Follow up } \\
\text { inspections }\end{array}$ & $\begin{array}{c}\text { Visits before } \\
\text { registration }\end{array}$ \\
\hline Pharmacies & 961 & 48 & 62 \\
\hline
\end{tabular}

Figures above relate to inspection activity between 1 April 2018 and 30 June 2018.

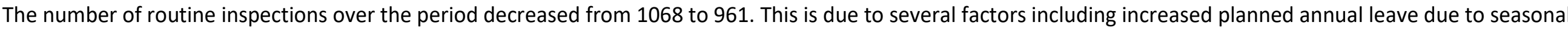

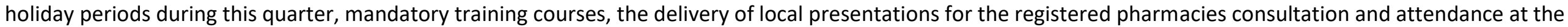
annual joint Conference with the CQC.

\subsection{Pharmacy premises not inspected}

\begin{tabular}{|c|c|c|c|c|c|c|}
\hline \multirow{2}{*}{\multicolumn{2}{|c|}{ Months since previous inspection }} & \multicolumn{4}{|c|}{$2017 / 18$} & \multirow{3}{*}{$\begin{array}{c}\text { 2018/19 } \\
\text { Q1 } \\
591\end{array}$} \\
\hline & & \multirow{2}{*}{$\begin{array}{l}\text { Q1 } \\
569\end{array}$} & \multirow{2}{*}{$\frac{\text { Q2 }}{505}$} & \multirow{2}{*}{$\begin{array}{c}\text { Q3 } \\
627 \\
\end{array}$} & \multirow{2}{*}{$\frac{\mathrm{Q4}}{\mathrm{734}}$} & \\
\hline & No. & & & & & \\
\hline 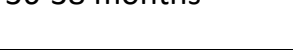 & $\%$ & $14.95 \%$ & $14.46 \%$ & $19.50 \%$ & $25.02 \%$ & $20.69 \%$ \\
\hline \multirow{2}{*}{$39-41$ months } & No. & 441 & 558 & 510 & 615 & 718 \\
\hline & $\%$ & $11.58 \%$ & $15.97 \%$ & $15.86 \%$ & $20.96 \%$ & $25.14 \%$ \\
\hline \multirow{2}{*}{$42-47$ months } & No. & 1,655 & 1,004 & 984 & 986 & 1014 \\
\hline & $\%$ & $43.47 \%$ & $28.74 \%$ & $30.60 \%$ & $33.61 \%$ & $35.50 \%$ \\
\hline \multirow{2}{*}{48 months or more } & No. & 1,142 & 1,426 & 1,095 & 599 & 533 \\
\hline & $\%$ & $30.00 \%$ & $40.82 \%$ & $34.05 \%$ & $20.42 \%$ & $18.66 \%$ \\
\hline Total & No. & 3,807 & 3,493 & 3,216 & 2,934 & 2,856 \\
\hline
\end{tabular}




\begin{tabular}{|l|c|c|c|c|c|c|} 
& $\%$ & $100.00 \%$ & $100.00 \%$ & $100.00 \%$ & $100.00 \%$ & $100.00 \%$ \\
\hline \multirow{2}{*}{ Of all registered pharmacies } & No. & 14,399 & 14,404 & 14,417 & 14,348 & 14,332 \\
\cline { 2 - 7 } & $\%$ & $26.44 \%$ & $24.25 \%$ & $22.31 \%$ & $20.45 \%$ & $19.93 \%$ \\
\hline
\end{tabular}

Figures correct as at 30th June 2018

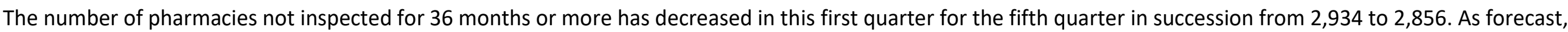

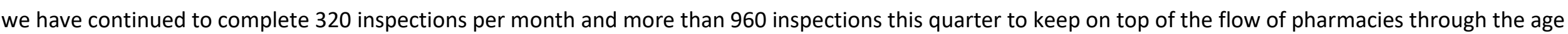
categories. There are no pharmacies in the sixty plus months category during this performance period. 
3.3 Age profile of pharmacies not inspected for $\mathbf{4 8}$ months and over

\begin{tabular}{|c|c|c|c|c|c|c|}
\hline $\begin{array}{l}\text { Months since previous } \\
\text { inspection }\end{array}$ & & East & North & South & West & Total \\
\hline \multirow{2}{*}{$48-50$ Months } & No. & 104 & 73 & 115 & 75 & 367 \\
\hline & $\%$ & $61.18 \%$ & $67.59 \%$ & $74.68 \%$ & $74.26 \%$ & $68.86 \%$ \\
\hline \multirow{2}{*}{$51-53$ Months } & No. & 49 & 34 & 37 & 22 & 142 \\
\hline & $\%$ & $28.82 \%$ & $31.48 \%$ & $24.03 \%$ & $21.78 \%$ & $26.64 \%$ \\
\hline \multirow{2}{*}{$54-59$ Months } & No. & 17 & 1 & 2 & 4 & 24 \\
\hline & $\%$ & $10.00 \%$ & $0.93 \%$ & $1.30 \%$ & $3.96 \%$ & $4.50 \%$ \\
\hline \multirow{2}{*}{ +60 Months } & No. & 0 & 0 & 0 & 0 & 0 \\
\hline & $\%$ & $0.00 \%$ & $0.00 \%$ & $0.00 \%$ & $0.00 \%$ & $0.00 \%$ \\
\hline \multirow{2}{*}{ Total } & No. & 170 & 108 & 154 & 101 & 533 \\
\hline & $\%$ & $100.00 \%$ & $100.00 \%$ & $100.00 \%$ & $100.00 \%$ & $100.00 \%$ \\
\hline
\end{tabular}

Figures correct as at 30th June 2018

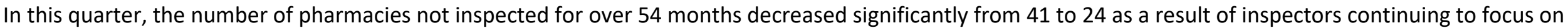

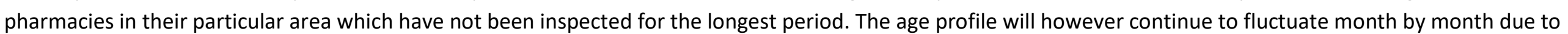

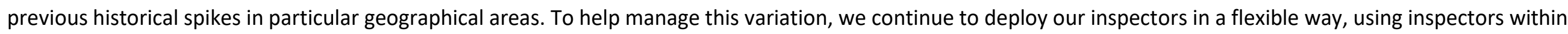
regions to assist colleagues in different areas, as well as across regions. 


\section{Top 5 standards ranked as not met}

\begin{tabular}{|c|l|c|}
\hline Standard no. & Description & Q4 Rank \\
\hline 4.3 & $\begin{array}{l}\text { Medicines and medical devices are: obtained from a reputable source; safe and fit for purpose; stored securely; } \\
\text { safeguarded from unauthorized access; supplied to the patient safely; and disposed of safely and securely }\end{array}$ & $\mathbf{4} 6$ \\
\hline 1.1 & The risks associated with providing pharmacy services are identified and managed & $\mathbf{1}$ \\
\hline 2.1 & $\begin{array}{l}\text { There are enough staff, suitably qualified and skilled, for the safe and effective provision of the pharmacy services } \\
\text { provided }\end{array}$ & $\mathbf{2 9}$ \\
\hline 4.2 & Pharmacy services are managed and delivered safely and effectively & 3 \\
\hline 1.2 & All necessary records for the safe provision of pharmacy services are kept and maintained & $\mathbf{2 9}$ \\
\hline
\end{tabular}

\subsection{Top 5 standards ranked as good}

\begin{tabular}{|c|c|c|c|}
\hline Standard no. & Description & & Q4 Rank \\
\hline 2.2 & $\begin{array}{l}\text { Staff have the appropriate skills, qualifications and competence for their role and the tasks they carry out, or are } \\
\text { working under the supervision of another person while they are in training }\end{array}$ & 299 & 1 \\
\hline 2.4 & There is a culture of openness, honesty and learning & 244 & 3 \\
\hline 1.2 & The safety and quality of pharmacy services are regularly reviewed and monitored & 243 & 2 \\
\hline 1.1 & The risks associated with providing pharmacy services are identified and managed & 181 & 4 \\
\hline 4.2 & Pharmacy services are managed and delivered safely and effectively & 179 & 5 \\
\hline
\end{tabular}

The above rankings relate to inspections carried out between 1 April 2018 and 30 June 2018 .

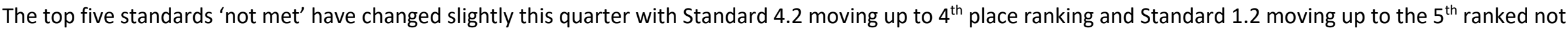

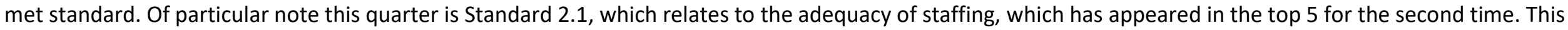

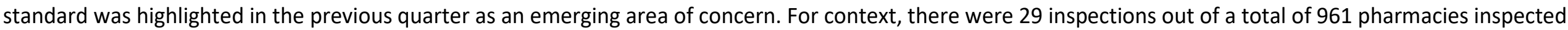

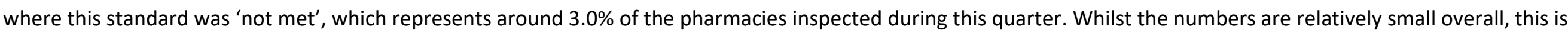

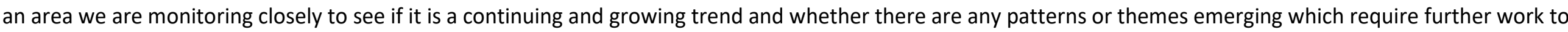

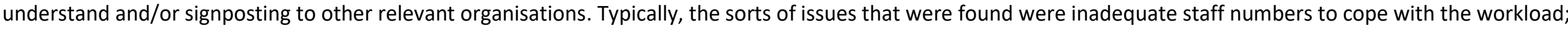

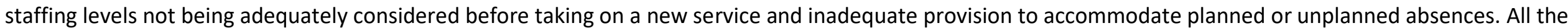
pharmacies concerned have had an improvement action plan to complete. 
The top five 'good' standards have remined relatively stable apart from 1.2 swapping places with 2.4 (is now in $3 \mathrm{rd}^{\text {th }}$ place).

\section{Complaints}

4.1 Formal complaints and negative feedback by category

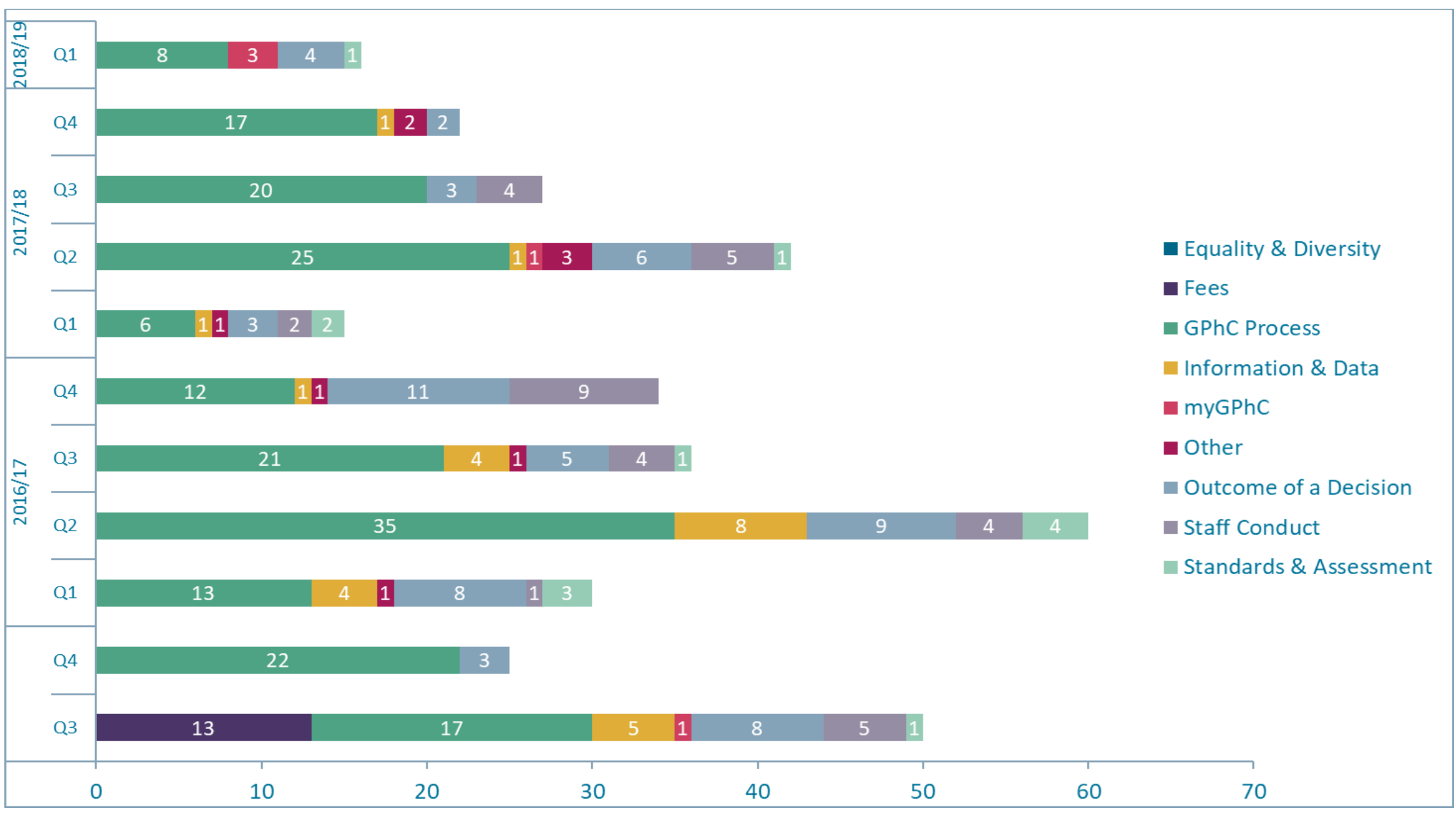


Figures correct as at 30th June 2018

\subsection{Formal complaints and negative feedback by category (cont.)}

In terms of the volume of complaints received, the first three months of this financial year sees a similar number to the previous year. 16 complaints were received in Q1 2018/19, compared to 15 in 2017/18. This number is below the 30 in 2016/17 and the 64 received in 2015/16. Of the 16 complaints received this year, one was upheld and another was partially upheld both related to GPhC processes.

There were no complaints regarding myGPhC in 2017/18, but with the launch of the new myGPhC portal for renewals and revalidation there have been three complaints in this Q1. None were upheld, but as the transition to online services continues an increase in complaints in Q2 regarding myGPhC should be anticipated. At the same time, having moved 54,789 users to the new system (as at 31 July), the receipt of only three complaints is a positive reflection on the work completed to date.

GPhC processes continued to attract the highest number of complaints in this reporting period, followed by outcome of a decision. Having seen a steady number of complaints relating to staff conduct since 2016/17, the absence of them now for two quarters in a row indicates a positive direction of travel. There is also now a nine-quarter trend with no complaints received relating to equality and diversity or fees.

Complaints provide valuable feedback and are viewed as positive agents for change. Themes or trends can be discerned, but on occasion issues can be identified from individual complaints. Currently, FtP receive quarterly analysis of complaints relating to their directorate and this will be rolled out to the other directorates in the near future. Going forward, the GPhC process category will be broken down even further to map out the source of complaint by process. This line of analysis is expected to produce additional insights, which will allow complaints to better drive business improvement in teams around the organisation. 


\section{Education}

\subsection{Accreditation and recognition activity}

\begin{tabular}{|c|c|c|c|c|c|c|c|c|c|c|}
\hline \multirow{2}{*}{ Course } & \multirow{2}{*}{ Type } & \multicolumn{2}{|c|}{ 2015-16 academic year } & \multicolumn{3}{|c|}{ 2016-17 academic year } & \multirow[b]{2}{*}{ Q2 } & \multicolumn{3}{|c|}{ 2017-18 academic year } \\
\hline & & Q1 & Q2 & Q3 & Q4 & $\bar{Q}$ 1 & & Q3 & $\overline{Q 4}$ & $\overline{\mathrm{Q} 1}$ \\
\hline \multirow{3}{*}{$\begin{array}{l}\text { Master of Pharmacy (MPharm) degree } \\
\text { 4-year }\end{array}$} & Accreditation & 2 & - & 1 & 4 & 4 & - & 1 & 1 & 2 \\
\hline & Reaccreditation & 2 & - & - & - & - & - & - & 1 & 6 \\
\hline & Interim visit & 0 & - & - & 5 & - & - & 1 & 5 & - \\
\hline \multirow{3}{*}{$\begin{array}{l}\text { Master of Pharmacy (MPharm) degree } \\
5 \text {-year integrated }\end{array}$} & Accreditation & - & - & - & - & - & - & - & 2 & 1 \\
\hline & Reaccreditation & - & - & - & - & - & - & - & - & - \\
\hline & Interim visit & - & - & - & - & - & - & - & - & - \\
\hline \multirow{2}{*}{$\begin{array}{l}\text { Master of Pharmacy (MPharm) degree } \\
2+2 \text { Overseas }\end{array}$} & Accreditation & - & - & - & - & - & - & - & - & 1 \\
\hline & Reaccreditation & - & - & - & - & - & - & - & - & 1 \\
\hline $\begin{array}{l}\text { Overseas pharmacist assessment } \\
\text { programme (OSPAP) }\end{array}$ & Reaccreditation & - & - & - & - & & - & - & 1 & 1 \\
\hline \multirow{3}{*}{ Independent prescribing } & Accreditation & 2 & - & 1 & 1 & 1 & - & 1 & - & 2 \\
\hline & Reaccreditation & 3 & - & 2 & 3 & 8 & 4 & 5 & 3 & 5 \\
\hline & Monitoring visit & 1 & - & 2 & - & 1 & 3 & 0 & - & 1 \\
\hline \multirow{2}{*}{$\begin{array}{l}\text { Level } 3 \text { Pharmacy technician } \\
\text { knowledge/competence }\end{array}$} & Approval/Accreditation & - & - & - & - & - & - & - & - & - \\
\hline & Reaccreditation & - & - & - & - & - & - & - & - & - \\
\hline \multirow{2}{*}{$\begin{array}{l}\text { Level } 2 \text { medicines counter assistant } \\
\text { and dispensing assistant }\end{array}$} & Accreditation & - & - & - & - & - & - & - & - & - \\
\hline & Reaccreditation & 1 & - & - & - & - & - & - & - & - \\
\hline
\end{tabular}

All events went ahead as scheduled. 
A high volume of events scheduled and have taken place for the 2017-18 academic year, particularly for MPharm degrees and independent prescribing programmes. The MPharm and OSPAP events would have taken place between January - July 2018, and prescribing events throughout the calendar year. The large number of events this academic year is due partly to natural peaks in the accreditation cycles but also to an increased interest from providers in provision of 5-year integrated MPharm degrees, and in the increase in the need for pharmacist prescribers which has led to increased funding for pharmacist prescribing programme places, resulting in interest from new course providers. 46 independent prescribing programmes are currently accredited. 


\section{Human Resources}

\subsection{Headcount Overview}

\begin{tabular}{|l|r|}
\hline GPhC & 30 June 2018 \\
\hline Headcount & $\mathbf{2 4 8}$ \\
\hline Permanent & 232 \\
\hline Fixed Term Contract & 16 \\
\hline Total Leavers & $\mathbf{1 7}$ \\
\hline Permanent leavers & 12 \\
\hline Turnover - Permanent (Apr-June) & $\mathbf{5 . 2 \%}$ \\
\hline Turnover - Permanent (for last twelve months: June 2017-June 2018) & $\mathbf{1 5 . 2 \%}$ \\
\hline Stability - Permanent staff & $\mathbf{8 5 \%}$ \\
\hline
\end{tabular}

The data above summarises the headcount position during the period of $01 / 04 / 18-30 / 06 / 18$. The total number of leavers for this period were 17 employees. The turnover rate excludes those employees who were on a fixed term contract.

The total number of voluntary leavers who had permanent contracts for this specific period equates to a turnover rate of $5.2 \%$. This is a favourable decrease from the $7.1 \%$ reported for the period (January - March 2018). The average rolling turnover rate for the 12-month period prior to $30 / 06 / 18$ is 15.2\%. This is a deterioration (from 13.8\%) for the 12-month period reported in the last HR report ${ }^{3}$.

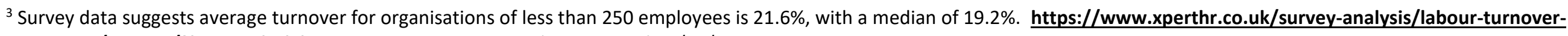
rates-2017/162496/?keywords=labour+turnover+rates+2017 last accessed 26/07/18
} 
The stability rate has been calculated based upon the number of permanent employees with more than 12 months employment at GPhC. On the $30^{\text {th }}$ June 2018, there were 187 permanent employees who had more than a 12-month employment period with GPhC. The stability percentage has increased from the previous reporting period (January to March 2018) from $81 \%$ to $85 \%$.

\subsection{Organisational Absence - Absence Percentages}

\begin{tabular}{|l|l|}
\hline Directorate & $\frac{\text { Absence \% }}{\text { Apr18 - Jun18 }}$ \\
\hline Organisation & $\mathbf{2 . 1 4 \%}$ \\
\hline Corporate Resources & $2.49 \%$ \\
\hline Education \& Standards & $1.78 \%$ \\
\hline Fitness to Practise & $3.34 \%$ \\
\hline Insight, Intelligence \& Inspection & $1.32 \%$ \\
\hline People & $1.13 \%$ \\
\hline
\end{tabular}

The table above details the absence percentages for the organisation and the individual Directorates at GPhC. In total 322 working days were lost due to absence in this period compared to a reported loss of 470 working days for the period January to March 2017 . The absence rate has fallen from $3.23 \%$ to $2.14 \%$.

Improvements in data collection have stemmed from a positive return process which now collates absence data more effectively, revealing greater accuracy in reported statistics. The launch of a 'Managing Attendance' policy will be supported by planned new management training is designed to tackle both long and short-term sickness absence. 
Absence rates by sector and organisation size, 2017 - \% of working time lost per annum:

\begin{tabular}{|l|l|l|l|}
\hline & & Broad economic sector: & Workforce size: \\
\hline & All & Public sector & $100-249$ \\
\hline Lower quartile & 3.6 & 7.1 & 2.8 \\
\hline Median & 5.6 & 9.8 & 4.3 \\
\hline Upper quartile & 7.8 & 11.0 & 6.4 \\
\hline Average & 6.1 & 9.7 & 5.2 \\
\hline $\begin{array}{l}\text { Note: } n=246 \\
\text { Source: XpertHR }\end{array}$ \\
\hline
\end{tabular}

Data taken from the CIPD Annual Survey Report 2017 - https://www.xperthr.co.uk/survey-analysis/sickness-absence-rates-and-costs-survey2018/163318/?keywords=sickness+absence+rates+costs+survey+2018 last accessed 26/07/18

\subsection{Employee Relations}

The table below is a summary of the employee relation cases by case type which were closed during the specified period:

\begin{tabular}{|l|l|}
\hline Case Type & No. of cases \\
\hline Total Cases & 3 \\
\hline Absence & 2 \\
\hline Grievance & 1 \\
\hline
\end{tabular}




\begin{tabular}{|l|l|}
\hline Performance & 0 \\
\hline Other & 0 \\
\hline
\end{tabular}

\subsection{Learning \& Development}

In Q1 L\&D provided a range of organisation-wide initiatives and support such as the GDPR e-learning, the online performance appraisal, Performance Development Review (PDR), and interactive communication workshops. L\&D also provided team specific support and coordination for sixteen delegates in Fitness to Practice and Inspection to complete the nationally accredited 'Advanced Professional Certificate in Investigative Practice'.

L\&D and Governance worked closely together to successfully launch the GDPR e-learning. All GPhC completed the training by the $18^{\text {th }}$ May and GDPR is now a mandatory training for new joiners in their induction. L\&D helped promote and encourage employees to undertake the eLearning, to build awareness of standardised data protection laws and new legislation.

The development of the online PDR was implemented in June. Moving away from paper-based, the customised system is user-friendly and allows employees to link their objectives with GPhC's Annual Plan, as well as having a personal development plan. HR and managers can now easily track and monitor progress. L\&D can review development plans and forecast future development needs. L\&D worked extensively throughout May to; develop a range of helpful resources, deliver presentations to each directorate and provide interactive workshops to help employees and people managers familiarise with the new system. Deadline to submit 2018 / 2019 objectives online is end of July and Q2 monitoring report shall feedback progress on PDR implementation.

To address one of the areas highlighted in the 2017 staff survey, an organisation-wide initiative throughout Q1 was the delivery of fourteen workshops 'Effective Communication Skills - Demonstrating Dignity \& Respect at work'. Ten workshops for employees and four workshops for people managers with 
a total of 143 delegates attending. We received positive feedback on both workshops with employees expressing their appreciation; recognising different communication styles and how they will be more mindful of how their behaviour impacts others. 


\section{General \\ Pharmaceutical \\ Council}

\section{Management Accounts 30 June 18}

The year to date position for the organisation is a variance of $£ 262 \mathrm{~K}$ against the "revised budget" including interest and tax.

The Actual year to date deficit is $£ 203 \mathrm{~K}$ versus a revised budget deficit of $£ 465 \mathrm{~K}$

Actual income is $₫ 5.9 \mathrm{M}$ year to date $(0.1 \%, £ 4 \mathrm{~K})$ below the revised budget

Actual overhead expenditure is $£ 6.1 \mathrm{M}(4.1 \%, £ 261 \mathrm{~K})$ below the revised budget

Income (See data table on page 3)

Income for the month of June 2018 , has an adverse variance of $0.5 \%$ ( $£ 12 \mathrm{~K}$ ) below the revised budget. This is due to Pharmacy Premises income for the month having an adverse variance of $4.17 \%$ ( $£ 13 \mathrm{~K}$ ) which mostly relates to new premises applications being lower than expected. Pre-registration income has a favourable variance for the month of $£ 21 \mathrm{~K}$ against the revised budget due to the number of candidates that applied to sit the exam were higher than anticipated by 115 applicants. The Year to date income variance is a marginal $0.1 \%(£ 4 \mathrm{~K})$ variance below the revised budget.

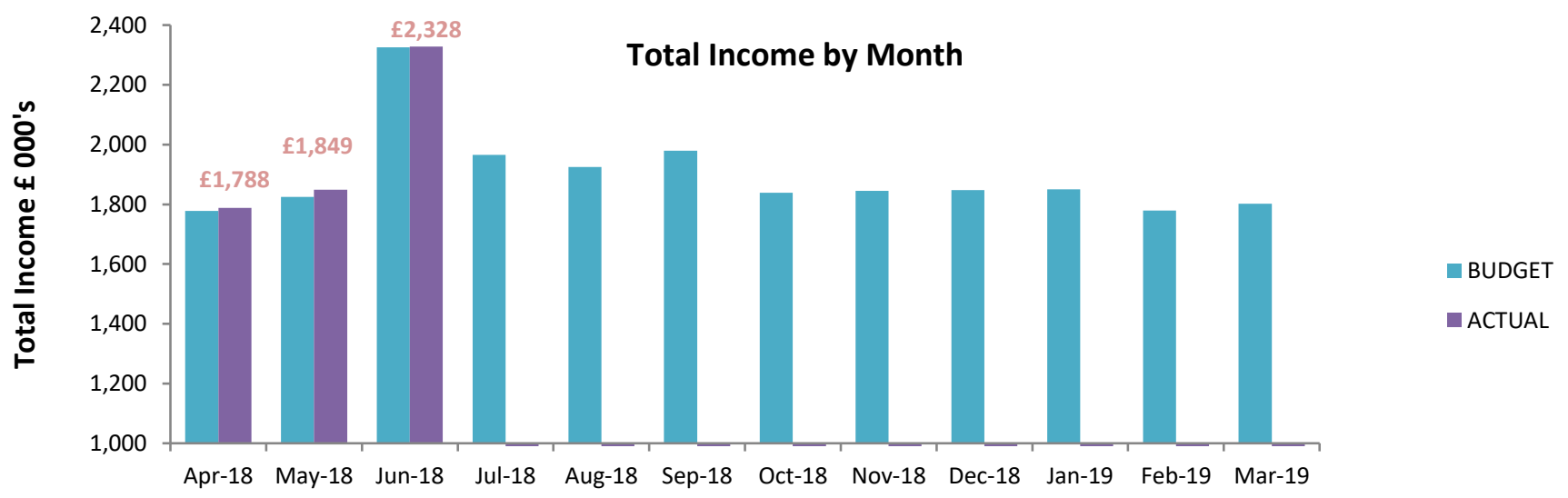

Iqbal Hussain

Page 1 of 5 
The accreditation event that was planned for June is now taking place in July which has reduced other income for the month by $43.8 \%$ ( $₫ 17 \mathrm{~K}$ ) below budget.

\section{Overheads (See data table on page 3)}

The organisation total year to date overheads expenditure has a favourable variance of $£ 260 \mathrm{~K}(4.1 \%)$ compared to the revised budget.

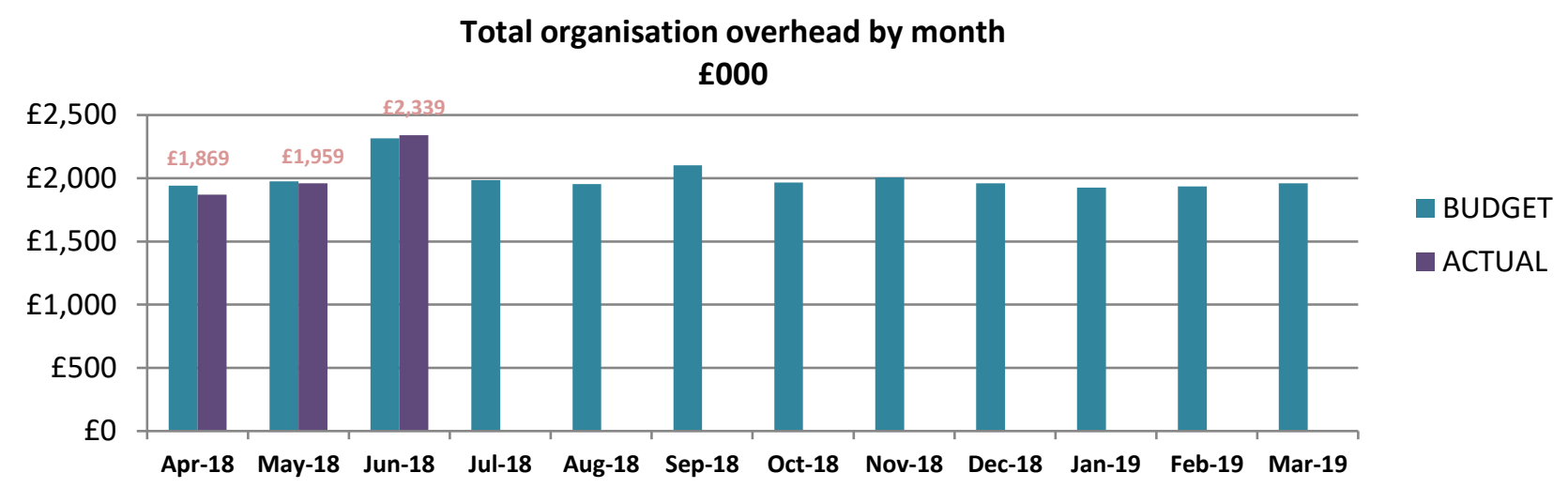

Employee costs year to date is $1.8 \%(£ 63)$ below the revised budget. This is mainly due to basic salaries which has a $4 \%$ vacancy saving. Contractors are $£ 47 \mathrm{~K}$ overspent year to date because temporary agency staff are being employed to cover permeant roles. Therefore, savings made in basic salaries would be offset against the overspend in contractors' costs. Recruitment has a favourable variance of $£ 14 \mathrm{~K}$ due to general recruitment delays partially arising from the budget challenge process which has now ended and recruitment should recommence in July 2018.

Council \& Associates actual expenditure variance against revised budget is marginally underspent year to date by $£ 1 \mathrm{~K}(0.2 \%)$.

Professional Fees year to date have a favourable variance of $8.4 \%$ ( $₫ 49 \mathrm{~K}$ ) against the revised budget. Consultancy costs are underspent year to date by $f 15 \mathrm{~K}$ due to Data \& Insight scoping strategy which has not yet commenced. Transcription costs are favourable by $£ 10 K$ due to a lower number of transcripts being requested and High Court legal costs are $£ 13 \mathrm{~K}$ underspent, as currently there is no judicial review pending.

Financial Costs are $£ 15 \mathrm{~K}(6.5 \%)$ year to date overspent against revised budget due to bank charges that relate to processing payment for exam fees and renewal fees. In addition, the loss of five laptops had to be written-off.

IT costs year to date are $33.2 \%$ (£154K) below budget due to IT development (New Cloud Project) work being delayed. 


\section{Summary Management Accounts 30 June 2018}

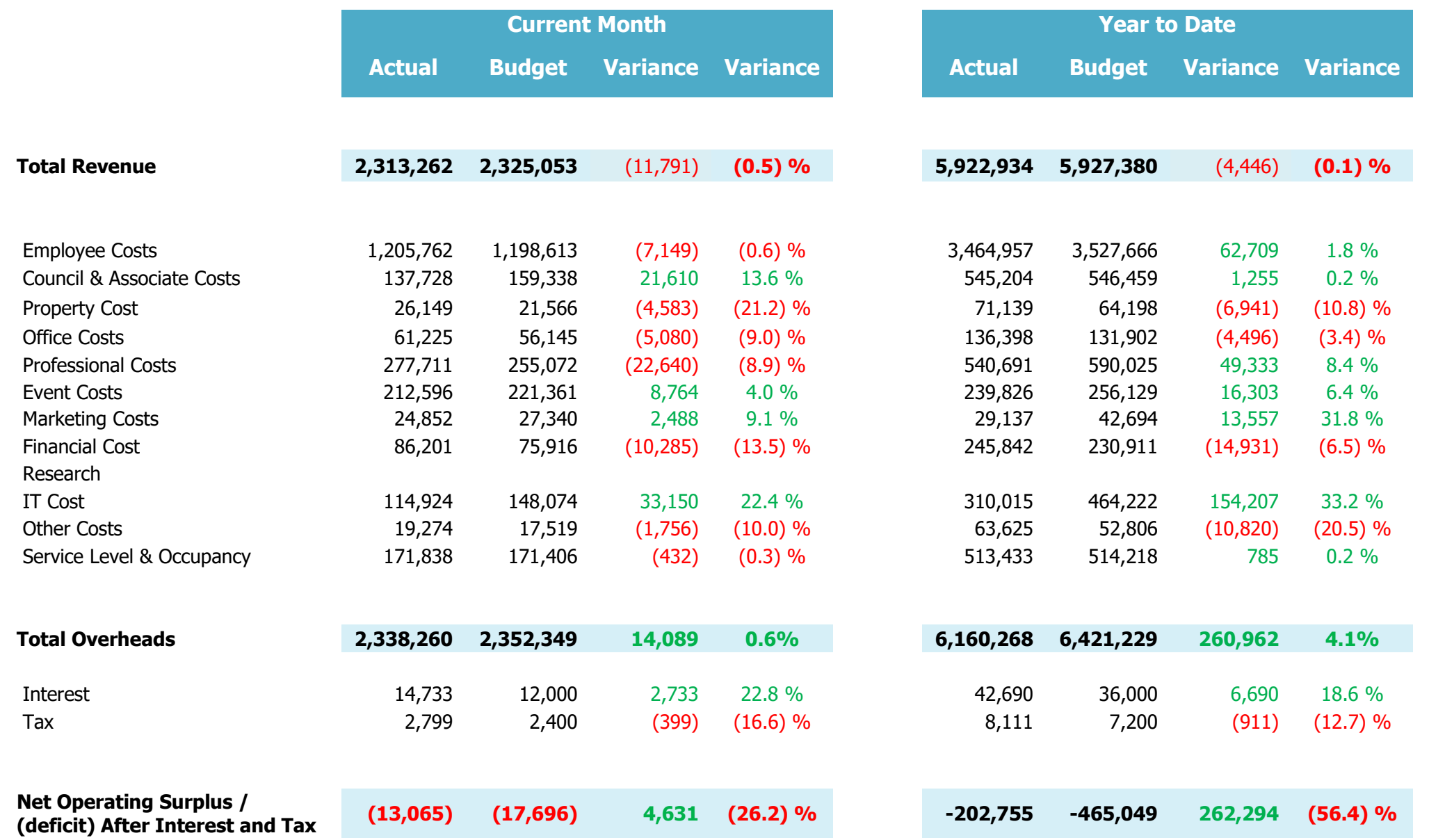


The STATEMENT OF FINANCIAL POSITION as at $\mathbf{3 0}$ June $\mathbf{1 8}$ shows a strong net position for the organisation.

$\begin{array}{rrr}\underline{30-J u n-18} & \underline{31 \text { Mar } 18} \\ \underline{£ 000} & \underline{000}\end{array}$

Fixed assets

Tangible assets

Intangible Assets

Investments

\begin{tabular}{rr}
3,625 & 3,804 \\
489 & 483 \\
12,500 & 12,500 \\
\hline 16,614 & 16,787 \\
\hline
\end{tabular}

\section{Current Assets}

Debtors

Bank and Cash

$\begin{array}{r}1,088 \\ 12,710 \\ \hline 13,798\end{array} \quad \begin{array}{r}13,814 \\ \hline 15,551\end{array}$

\section{Creditors}

Amounts failing due within one year

Net current assets

\section{Creditors}

Amounts failing due after more than one year Provision for liabilities

Total Net assets

\section{Funds employed}

Fixed Asset Reserve 


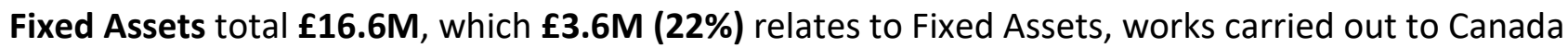
Square office and upgrading laptops for office based workers.

Intangible Assets such as Information Technology Development projects (Cloud Strategy, Revalidation, Case Tracker \& Registrant Online) equates to $\mathbf{f 4 8 9 K}$.

Investments are six months of expenditure $\mathbf{f 1 2 . 5 M}(\mathbf{7 8 \% )}$ that has been invested in long term deposits with various banks.

Current Assets f13.7M includes cash held in bank accounts most of which relates primarily to registrants' income. The debtors' figures include cost recovery for high court appeals as well as prepayments. The high court debtors balance will be adjusted at the end of each financial year to include bad debt provision. Prepayments figures includes amounts paid in advance for rent, annual licences and subscriptions.

Current Liabilities include deferred income in relation to fees paid in advance for all registrant groups and grant income for the building which will be released over the remaining term of the lease.

Long term Liabilities include the Landlord contribution to the office fit out which has been offset by the provision for future rent increases and dilapidation provision which has been added this year due to a recent review of the lease.

\section{Cash Balance}

Cash Balance 2018-19 vs 2017-18

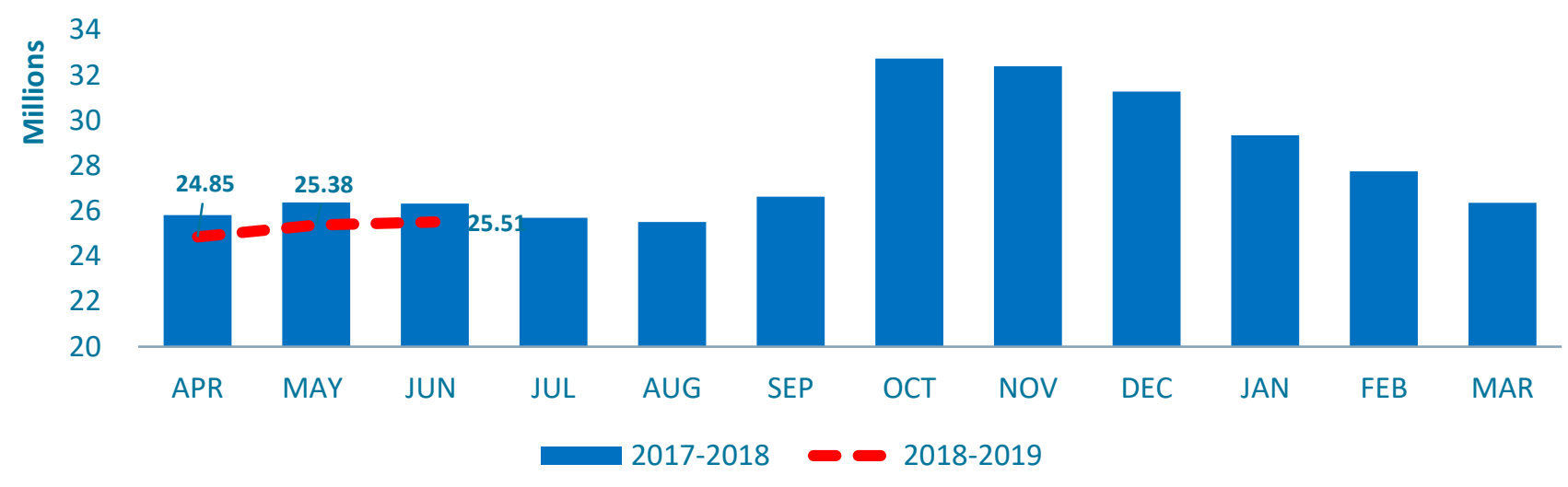

Over the last 3 months the cash balance has reduced to a lower level than the previous year at this same point in time. This is line with the organisation intention to increase expenditure in order deliver strategic objectives. The GPhC reserves are made up of General and Fixed Assets reserve. 
General

Pharmaceutical

Council

\section{Annual plan progress report 2018/19}

Quarter 1: April - June 2018 


\section{Introduction}

This report provides an update on the key strategic priorities in our Annual plan 2018/19.

The reporting period covers quarter 1, April to June 2018.

\section{Overview}

\begin{tabular}{|l|l|l|}
\hline Strategic Priorities & Status & $\begin{array}{l}\text { Direction } \\
\text { of travel }\end{array}$ \\
\hline Building our data, information, intelligence and insight capability & G & \\
\hline Developing our approach to fitness to practise & A & $\begin{array}{l}\text { New } \\
\text { Strategic } \\
\text { Priority }\end{array}$ \\
\hline $\begin{array}{l}\text { Securing assurance and promoting improvement in registered } \\
\text { pharmacies }\end{array}$ & G & \\
\hline $\begin{array}{l}\text { Improving standards of care through regulation of education and } \\
\text { training }\end{array}$ & A & \\
\hline Transforming our organisation, our services and processes & A & \\
\hline
\end{tabular}

Key
\begin{tabular}{|c|l|}
\hline $\begin{array}{c}\text { Status/direction } \\
\text { of travel }\end{array}$ & Definition \\
\hline R & $\begin{array}{l}\text { Significant issues, aims } \\
\text { may not be metto } \\
\text { time/budget }\end{array}$ \\
\hline A & $\begin{array}{l}\text { Some issues emerring, } \\
\text { aims still achievable }\end{array}$ \\
\hline G & On track/completed \\
\hline B & Not started \\
\hline 1 & $\begin{array}{l}\text { Rating Improved from last } \\
\text { period }\end{array}$ \\
\hline$\square$ & $\begin{array}{l}\text { Rating worsened from last } \\
\text { period }\end{array}$ \\
\hline$\Rightarrow$ & $\begin{array}{l}\text { Rating from last period } \\
\text { unchanged }\end{array}$ \\
\hline & \\
\hline
\end{tabular}




\section{Building on our data, information, intelligence and insight capability}

\section{Strategic aim:}

- The pharmacy team have the necessary knowledge, attitudes and behaviours

- Registered pharmacies deliver safe, effective care and services

- Pharmacy regulation is efficient and effective

\section{In 2018/19 we will:}

- Develop a phased insights and intelligence strategy to improve our capacity and capability to report, learn from and act on our work more efficiently

- Improve the quality and consistency of the data that we hold for key statutory and governance performance reports and the supporting data infrastructure

- Share the insights from what we have learned from inspections of registered pharmacies to date

- Conduct a registrant workforce survey to inform our work and that of stakeholders

- Continue to update our data approach and procedures to ensure compliance with the General Data Protection Regulation (GDPR)

- Create the development framework for a pilot organisation wide enquiry hub to receive, co-ordinate and analyse all incoming information and concerns to inform more proactive and tailored regulatory responses at an earlier stage

\section{Key links and assumptions}

- Resources for all business teams are available to do this work and teams will work collaboratively with support from senior leaders and managers

- The volume of data and information requests remains stable so that there is capacity to do this work

- Partner organisations must be engaged and have resources

\section{What does success look like?}

- A clear insights and intelligence strategy in place guiding our priority areas for action

- Standardised and automated reporting of statutory and governance performance reports

- Published insights from inspections of registered pharmacies are being used to inform and drive improvements in pharmacy practice

- Up to date baseline established of what pharmacy professionals are doing and where

- No personal data breaches reportable to the Information Commissioner's Office

- Information rights requests responded to appropriately and within time limits

- Overall development framework in place with phase one pilot of an enquiry hub (enhanced triage) in Fitness to Practise up and running; (this pilot is being reported under 'Developing our approach to Fitness to Practise')

\section{Main risks}

- If resources (capacity and capability) are not available, work will take longer to complete 


\section{Outline timetable:}

\begin{tabular}{|c|c|c|c|}
\hline April-June 2018 & July-September 2018 & October-December 2018 & January-March 2019 \\
\hline $\begin{array}{l}\text { Complete baseline mapping of } \\
\text { organisation to inform the } \\
\text { Insights and Intelligence strategy } \\
\text { - Commence end to end review } \\
\text { and design for the automation of } \\
\text { the production of key statutory } \\
\text { and governance reports } \\
\text { - Commission research on what we } \\
\text { have learned from our current } \\
\text { approach to inspecting registered } \\
\text { pharmacies } \\
\text { - GDPR e-learning training sourced } \\
\text { and implemented } \\
\text { - Internal data protection policies } \\
\text { and procedures updated } \\
\text { Website and myGPhC privacy } \\
\text { policies updated } \\
\text { Consultation on publication and } \\
\text { disclosure policy begins } \\
\text { New data protection contract } \\
\text { clauses and schedules sent to } \\
\text { suppliers of priority contracts }\end{array}$ & $\begin{array}{l}\text { - Complete standardisation and } \\
\text { automation of Professional } \\
\text { Standards Authority (PSA) data } \\
\text { sets } \\
\text { - Commence development of } \\
\text { automated Council Performance } \\
\text { Monitoring Reports } \\
\text { - Begin research on learning from } \\
\text { our inspections of registered } \\
\text { pharmacies to date } \\
\text { - Scope requirements for a } \\
\text { registrant survey } \\
\text { - Supplier contract GDPR variations } \\
\text { completed } \\
\text { - Further tailored GDPR training for } \\
\text { key functional areas developed } \\
\text { and dates planned }\end{array}$ & $\begin{array}{l}\text { - Insights and intelligence strategy } \\
\text { presented to Council including } \\
\text { priority areas of focus with plan } \\
\text { - } \text { Operational data warehouse } \\
\text { developed to include addition of } \\
\text { datasets } \\
\text { - Research on learning from } \\
\text { inspections finalised } \\
\text { - Identify options to conduct and } \\
\text { analyse registrant survey } \\
\text { - Publication and disclosure policy } \\
\text { finalised } \\
\text { - Personal data processing records } \\
\text { reviewed and updated } \\
\text { - Further GDPR tailored training } \\
\text { launched } \\
\text { Development framework for a } \\
\text { pilot organisation wide enquiry } \\
\text { hub completed }\end{array}$ & $\begin{array}{l}\text { - Continued standardisation and } \\
\text { consolidation activities for data } \\
\text { - Conduct registrant survey } \\
\text { - GDPR compliant information } \\
\text { sharing agreements in place for } \\
\text { key Memorandum of } \\
\text { Understanding (MoU) partners } \\
\text { - Tailored GDPR training continues } \\
\text { - Develop a public perceptions } \\
\text { survey }\end{array}$ \\
\hline
\end{tabular}




\section{Commentary for Q1:}

\section{Data, Insights and Intelligence}

In this quarter we have established a baseline of what data sources exist across the organisation, providing us with a picture of the end to end flow of data (inputs, processes and outputs). This has informed the framework for the Insights and Intelligence Strategy. In addition, work to standardise and automate the PSA data set is underway, including creation of revised data dictionaries, documenting data fields with business definitions and the building of a new operational reporting database for sign off with business areas. This work will contribute to improving the quality and consistency of the data we hold, our data infrastructure and the efficiency in our reporting capability.

Commissioning of research on learning from our inspections of registered pharmacies commenced during this quarter. In addition, scoping of requirements for a registrant survey started, including conversations with key stakeholders.

\section{GDPR Compliance}

The GDPR and Data Protection Act 2018 came into force on 25 May 2018. Work in the first quarter focussed on the launch of an updated set of data protection and privacy policies, a review of supplier contracts and staff awareness. The consultation on the publication and disclosure policy was launched in June and closes in September. 95\% of staff and associates had completed GDPR e-learning by 25 May and further training is being planned to start in the Autumn.

The overall RAG status remains green because activities are progressing in line with the previously updated scope of work and timelines. 


\section{Developing our approach to fitness to practise}

Strategic aim: The pharmacy team have the necessary knowledge, attitudes and behaviours

In 2018/19 we will:

- Develop a strategy for the future of fitness to practise (FtP) that is focused on protecting the public whilst being more restorative and less adversarial in our approach. We will draft a consultation document to support this

- Undertake and evaluate pilots to inform the development of the strategy including an enquiry stage (enhanced triage) and the use of pre-Investigating Committee undertakings

- Improve how we categorise the concerns we receive to better understand our caseload and draw out any insights for the strategy development

- Ensure that the future FtP strategy reflects the learning from the health professions sector and recent inquiries and reports

- Undertake the planning for an external review which will look at the links between our fitness to practise processes and the mental health implications for those involved, including registrants, complainants and witnesses. The review will be carried out in 2019/20

\section{What does success look like?}

- The draft future FtP strategy is drafted and ready for consultation

- The draft strategy clearly sets out our ambition; always taking prompt action on serious concerns and being proportionate, fair and timely in the use of regulatory powers.

- Our developing draft fitness to practise (FtP) strategy is informed by wider sector learning, including from the Williams review, Gosport inquiry, other regulatory reviews and other regulators as well as from our own internal pilots and developments

- We will have a clear plan for the delivery of the external review. The plan will include how pharmacy sector stakeholders are to be involved and some of the activities

\section{Key links and assumptions}

- We will have a public consultation on the future fitness to practise strategy which will incorporate aspects of the work on assessing the impact on mental health

\section{Main risks}

- Availability of review panel members to engage with the planning of the mental health and FtP review

- Limited resources in terms of capacity to complete the ongoing work as well as delivering the regulatory function 
Outline timetable:

\begin{tabular}{|c|c|c|c|}
\hline April-June 2018 & July-September 2018 & October-December 2018 & January-February 2019 \\
\hline $\begin{array}{l}\text { Develop draft strategy and key } \\
\text { associated initiatives } \\
\text { - Background work on mental } \\
\text { health and FtP including } \\
\text { reviewing work undertaken by } \\
\text { other regulators } \\
\text { - Research and planning on } \\
\text { planned pilots and improvements } \\
\text { to categorisation }\end{array}$ & $\begin{array}{l}\text { - Consultation approach agreed with } \\
\text { Senior Leadership Group } \\
\text { - Enquiry and Pre-Investigating } \\
\text { Committee undertakings pilot } \\
\text { launched } \\
\text { - Agree terms of reference and } \\
\text { glossary for mental health and FtP } \\
\text { - Appoint review panel for mental } \\
\text { health and FtP }\end{array}$ & $\begin{array}{l}\text { - Review process and identify key } \\
\text { changes that can be made in- } \\
\text { house } \\
\text { - Launch revised categories } \\
\text { - Evaluation of pilots and strategy } \\
\text { development } \\
\text { - Plan stakeholder event for mental } \\
\text { health and FtP }\end{array}$ & $\begin{array}{l}\text { - Implement any internal } \\
\text { recommendations } \\
\text { - Plan consultation for launch in } \\
\text { early } 2019 / 20\end{array}$ \\
\hline
\end{tabular}




\section{Commentary for Q1:}

\section{Future fitness to practise}

During this quarter we have:

- drafted plans for an enquiry stage (enhanced triage) pilot to commence in the Autumn (engaging with policy colleagues)

- developed revised fitness to practise categories in final draft, taking account of Professional Standards Authority research and in collaboration with data and policy colleagues

- developed an action plan that will enable us to take forward the FtP recommendations of the recent PSA Lessons Learned and the Williams reviews (a plan to address the Gosport Inquiry recommendations will follow in Autumn)

- met with the Nursing and Midwifery Council on their future fitness to practise approach and development process

\section{Mental Health}

During this quarter we have:

- set out the scope, governance and delivery of the work in the draft terms of reference

- developed a draft glossary of terms to be used throughout the project

- engaged with key stakeholders and project partners including Pharmacist Support, Pharmacist Defence Association and the Nursing and Midwifery Council

- identified the majority of the review panel members (patient representative or patient representative body to be recruited)

The overall RAG status is amber. The activities in quarter 1 have been delivered on time but the planned work could be subject to delay due to the resources available to initiate and deliver these projects. 


\section{Securing assurance and promoting improvement in registered pharmacies}

Strategic aim: The pharmacy team have the necessary knowledge, attitudes and behaviours

\begin{tabular}{|c|c|}
\hline RAG & Direction of travel \\
\hline $\mathrm{G}$ & \\
\hline
\end{tabular}

\section{In 2018/19 we will:}

- Agree with government a timetable for commencing new powers to publish inspection reports, and share learning to promote improvement

- Consult on and implement our approach to publication of inspection reports and our updated proposals on pharmacy inspection

- Implement new enforcement powers to ensure our standards are met in registered pharmacies

- Build understanding among our stakeholders of our powers and tools for regulating both individual members of professions and pharmacy owners

- Issue guidance to pharmacy owners to support safe and effective care by all staff within the pharmacy team

\section{Key links and assumptions}

- Publication of inspection reports by the end of March 2019 is dependent upon approval of the refined inspection approach in December 2018 and the build of the reporting website with supporting infrastructure

\section{What does success look like?}

- Outcomes of inspections of registered pharmacies are easily accessible and transparent to members of the public, the pharmacy sector and other stakeholders, providing assurance and driving continuous improvements in the quality of pharmacy practice

- Stakeholders are clear on how their views informed the approved approach to regulating registered pharmacies

- The sharing of insights from inspections of registered pharmacies are used to inform improvements in the sector

- Pharmacy owners are clear on how our enforcement powers will be applied when standards are not met

- Pharmacy owners are clear what the regulator's expectations are for a safe and effective pharmacy team

- Key stakeholders have a basic understanding of our role, powers and tools for regulating professionals and owners

\section{Main risks}

- Until the outcome of the consultation is available, it is unclear whether our proposals will draw broad support from the public and the sector

- The development of the IT infrastructure is the key building block for the publication and implementation of the refined inspection approach within the current timelines

- Limited external interest in, or engagement with, our work among key stakeholders at a time of significant challenge within pharmacy, health and government 


\section{Outline timetable:}

\begin{tabular}{|c|c|c|c|}
\hline April-June 2018 & July-September 2018 & October-December 2018 & January-March 2019 \\
\hline $\begin{array}{l}\text { - Worked closely with the } \\
\text { Government to agree the } \\
\text { timetable for commencing the } \\
\text { Pharmacy (Premises Standards, } \\
\text { Information Obligations etc) Order } \\
2016 \\
\text { - Guidance for pharmacy owners to } \\
\text { ensure a safe and effective } \\
\text { pharmacy team published and } \\
\text { promoted to all owners and } \\
\text { pharmacy professionals, to raise } \\
\text { awareness of what is expected of } \\
\text { pharmacy owners } \\
\text { - Consultation on developing our } \\
\text { approach to regulating registered } \\
\text { pharmacies published } \\
\text { - The consultation included a } \\
\text { summary of our new enforcement } \\
\text { powers and the principles of our } \\
\text { approach to enforcement which } \\
\text { will help increase stakeholder } \\
\text { understanding of our powers and } \\
\text { tools } \\
\text { - Commissioned research to inform } \\
\text { our approach to registered } \\
\text { pharmacies and drive } \\
\text { improvement in the sector }\end{array}$ & $\begin{array}{l}\text { - Consultation on developing our } \\
\text { approach to regulating registered } \\
\text { pharmacies closes } \\
\text { - Research begins on what we have } \\
\text { learned from our current approach } \\
\text { to inspecting registered pharmacies } \\
\text { - Develop new enforcement policy, } \\
\text { engaging with key stakeholders to } \\
\text { help inform the policy and increase } \\
\text { their awareness of our powers and } \\
\text { tools } \\
\text { - Publish discussion paper on new } \\
\text { proposals to protect people trying } \\
\text { to obtain medicines online, setting } \\
\text { out our powers and tools to } \\
\text { regulate online pharmacies } \\
\text { - Hold meetings with ministers and } \\
\text { other key parliamentarians in three } \\
\text { countries of GB, and with leading } \\
\text { patient organisations, to explain } \\
\text { our role, powers and tools for } \\
\text { regulation }\end{array}$ & $\begin{array}{l}\text { - Analysis of data and publication of } \\
\text { research on what we have learned } \\
\text { from our current approach to } \\
\text { inspecting registered pharmacies } \\
\text { - Analysis report of our approach to } \\
\text { regulating registered pharmacies } \\
\text { shared with Council and published } \\
\text { - Approach to publication and the } \\
\text { way we inspect registered } \\
\text { pharmacies agreed with Council; } \\
\text { promotion of new approach to all } \\
\text { key stakeholders to build } \\
\text { understanding and awareness } \\
\text { - Publish our new enforcement policy } \\
\text { - Publish updated guidance on } \\
\text { supplying medicines at a distance } \\
\text { and use publication as an } \\
\text { opportunity to explain our role and } \\
\text { how we work with other regulators } \\
\text { in this area }\end{array}$ & $\begin{array}{l}\text { - Start implementation activities for } \\
\text { the publication of inspection } \\
\text { reports and agreed refinements to } \\
\text { our approach to inspection } \\
\text { - Promote publication of inspection } \\
\text { reports to all key stakeholders to } \\
\text { help increase awareness of our } \\
\text { role in inspecting pharmacies and } \\
\text { new publication powers }\end{array}$ \\
\hline
\end{tabular}


- Developed new public affairs

strategy and taken forward a series

of meetings with parliamentarians

and other stakeholders to build

understanding and awareness of

our role

\section{Commentary for Q1:}

Agree with government a timetable for commencing new powers to publish inspection reports, and share learning to promote improvement

On 24 May 2018, the Pharmacy (Premises Standards, Information Obligations etc) Order came into force, providing us with the power to publish

inspection reports and wider powers of enforcement against the premises standards.

We are continuing our work to share what we have learned from inspection. Since we introduced the new 'show and tell' approach to inspections in 2013 , we have inspected more than 13,000 registered pharmacies across Great Britain and we are in the process of commissioning external research to review and evaluate what we have learned from inspection during this time.

\section{Consult on and implement our approach to publication of inspection reports and our updated proposals on pharmacy inspection}

In May 2018, we launched the public consultation on developing our approach to regulating registered pharmacies, which is set to run until 9 August 2018. The refined approach to inspection focuses on 6 main areas: changes to the types of inspections; moving to unannounced inspections; changing inspection outcomes; requiring all standards to be met to receive an overall 'standards met' outcome; publishing inspection reports; and sharing examples of notable practice in a 'knowledge hub'. We have held three stakeholder engagement events in Edinburgh, Cardiff and London together with 22 local events led by inspectors.

\section{Implement new enforcement powers to ensure our standards are met in registered pharmacies}

The Pharmacy (Premises Standards, Information Obligations etc) Order 2016 brings about a number of changes to our enforcement powers. The effective use of these powers in regulation is important to secure compliance with our standards for registered pharmacies and, where necessary, to ensure that those who have not complied may be held to account. We are developing an enforcement policy, which sets out our approach to enforcement when a pharmacy is found not to be meeting our standards. This will be used to ensure proportionate and consistent decision making about when to use our enforcement powers.

\section{Guidance to pharmacy owners to support safe and effective care by all staff within the pharmacy team}

At the Council meeting in May new guidance for pharmacy owners on ensuring a safe and effective pharmacy team was agreed and published shortly afterwards. At the same meeting, Council also agreed with the recommendation to take forward the education-related work for unregistered pharmacy staff. 


\section{Discussion paper on making sure patients can obtain medicines and other pharmacy services safely online}

In June a discussion paper which proposed some additions to our guidance on supplying pharmacy services at a distance was published. This highlighted areas we were proposing to strengthen including safeguards for patients trying to obtain medicines online. The discussion paper explained our role and powers to regulate online pharmacies, and how we work with other regulators which also have a role in regulating online primary care services.

The overall RAG rating and direction of travel for the key pieces of work associated with the delivery of this priority area remains the same as the previous quarter. This reflects the good progress made against the actions planned for this quarter, the majority of which cover the continuation of work already underway. 


\section{Improving standards of care through regulation of education and training}

Strategic aim: The pharmacy team have the necessary knowledge, attitudes and behaviours

\section{In 2018/19 we will:}

- Implement our new revalidation framework to provide assurance that pharmacy professionals continue to meet the required standards of professionalism throughout their careers

- Implement new standards for the initial education and training for pharmacy technicians working with course developers and providers

- Consult on, review and agree new standards for pharmacist independent prescribers followed by implementation activities with course developers and providers

- Initiate our work to review and consult on initial education and training for pharmacists so that initial education provided will meet the future needs of the public in relation to pharmacy services

\section{Key links and assumptions}

- Stakeholders will support direction of travel for new pharmacist initial education training standards

- Courses based on new initial education and training standards for pharmacy technicians will be ready for accreditation at the end of 2018/start of 2019 so they are ready for delivery in September 2019

- Courses based on new education and training standards for pharmacist independent prescribers are ready for accreditation in early 2019

\section{What does success look like?}

- Registrants will be able to renew and submit their revalidation records to demonstrate more effectively they are keeping up-to-date and reflecting on the benefit of their learning and practice on the people who use their services

- Revised standards for education and training for pharmacy technicians, pharmacist independent prescribers and pharmacists provide assurance that pharmacy professionals are fully equipped to play a leading role in the future of multi-professional healthcare

- Course providers for pharmacy technicians, pharmacist independent prescribers and pharmacists meet our new standards effectively through our accreditation or recognition processes

\section{Main risks}

- Late sign up for the new version of MyGPhC

- Our proposals for pharmacist IET standards that cannot be delivered due to opposition from stakeholders 


\section{Outline timetable:}

\begin{tabular}{|c|c|c|c|}
\hline April-June 2018 & July-September 2018 & October-December 2018 & January-March 2019 \\
\hline $\begin{array}{l}\text { - Revalidation: Further revalidation } \\
\text { guidance and examples published } \\
\text { - Education: Analyse responses to } \\
\text { pharmacist independent } \\
\text { prescribing standards } \\
\text { consultation } \\
\text { - Education: Engagement with } \\
\text { pharmacy technician course } \\
\text { developers and providers } \\
\text { - Education: Engagement with } \\
\text { three new working groups for the } \\
\text { development of the pharmacist } \\
\text { IET standards }\end{array}$ & $\begin{array}{l}\text { - Revalidation: The next phase of } \\
\text { revalidation development to go live } \\
\text { - Education: Workshops on elements } \\
\text { of pharmacist IET standards } \\
\text { - Education: Prepare papers for } \\
\text { Council on pharmacist independent } \\
\text { prescribing } \\
\text { - Education: Continued engagement } \\
\text { with pharmacy technician course } \\
\text { developers and providers }\end{array}$ & $\begin{array}{l}\text { - Revalidation: Recruitment and } \\
\text { selection of revalidation reviewers } \\
\text { - Education: Launch pharmacist IET } \\
\text { standards consultation } \\
\text { - Education: Put proposal for policy } \\
\text { on non-registered pharmacy staff to } \\
\text { Council } \\
\text { - Education: Accredit new courses } \\
\text { based on pharmacy technician IET } \\
\text { standards } \\
\text { - Education: Agree revised IET } \\
\text { standards for pharmacist } \\
\text { independent prescribers following } \\
\text { consultation } \\
\text { - Education: Present revised } \\
\text { proposals for the education and } \\
\text { training of non-registered pharmacy } \\
\text { staff }\end{array}$ & $\begin{array}{l}\text { - Revalidation: Preparation for first } \\
\text { revalidation reviews } \\
\text { - Revalidation: The next phase of } \\
\text { revalidation goes live. } \\
\text { - Revalidation: Council review of } \\
\text { evaluation approach for } \\
\text { revalidation } \\
\text { - Education: Begin analysis of } \\
\text { pharmacist IET standards } \\
\text { consultation } \\
\text { - Education: Begin accreditation of } \\
\text { courses based on new Education } \\
\text { and Training (ET) standards for } \\
\text { pharmacist independent } \\
\text { prescribers }\end{array}$ \\
\hline
\end{tabular}

\section{Commentary for Q1:}

Revalidation: MyGPhC and the new recording format for revalidation records launched successfully in April. Further guidance for peer discussion and examples of good records have been published to assist registrants to meet the requirements of revalidation for pharmacy professionals. The next phase of the development to our systems and processes has commenced. We are on track to release a new functionality to allow registrants to submit their revalidation records to us for the first time in September 2018.

Pharmacy technician IET standards: These standards are being used by course developers to create new courses and we are in contact with relevant stakeholders in England, Scotland and Wales about this. We expect courses to be put forward for accreditation in the Autumn of 2018 and are ready to do this. 
Pharmacist independent prescriber ET standards: The consultation on these standards has now closed and analysis of the consultation responses is complete. We had 340 responses from individuals and 59 organisational responses. As was the case for the pharmacy technician IET standards, we will provide an evidence framework with guidance for course developers. We are progressing on our target to share the consultation analysis report in September 2018 and revised standards after that. Also in September we plan to provide Council with a more wide-ranging paper about pharmacist independent prescribing.

Education and training of non-registered pharmacy staff: In response to feedback to our 2017 consultation on the education and training of non-registered pharmacy staff, we have been developing our thinking on this subject and will be returning to Council with revised proposals in Autumn 2018.

Pharmacist IET standards: Our work on these standards is continuing. To move standards drafting forward we have convened three working groups, to look at 1. learning outcomes, 2. the place of prescribing in IET and 3. pre-registration training standards to feed into the development of learning outcomes and standards so they are ready for consultation.

The RAG status remains amber as the work programme is extensive, highly complex and now also includes the revalidation and unregistered pharmacy team work. We have also accelerated the timescale for consulting on the revised initial education and training standards for pharmacists meaning there are some complex issues to resolve fully in a slightly shorter time frame. 
Transforming our organisation, our services and processes

Strategic aim: Pharmacy regulation is efficient and effective

\section{In 2018/19 we will:}

- Improve online services to enable registrants to complete and review their revalidation records online

- Improve online services for registration, renewal and application functions in phases throughout the year

- Embed equality, diversity and inclusion (EDI) in both our role as a regulator and employer

- Conduct a survey of our registrant's views of the GPhC's services and communications in order to identify areas for further improvement

- Invest in updating our culture, ways of working and means of holding ourselves to account so that we have the right staff with the right skills and attitudes to adapt to the evolving world of regulation and pharmacy professionals

- Continue to invest in our IT infrastructure and applications by moving to for future needs

- Align our risk management approach to the ISO31000 standard cloud based solutions in order to provide a flexible and robust foundation

What does success look like?

- Registrants can complete and submit their revalidation records online and we can review their records online

- Registrant services are improved with new online services for registration, renewal and application functions

- The GPhC will progress commitments to EDI. For one of our key priority areas, disability, we will have started the implementation of the formal disability standard

- The registrant survey findings inform the baseline against which we measure any improvement in our communications and services

- The culture statement, refreshed values, and behaviours are embedded in every part of the GPhC and used by managers and staff to underpin the Performance Development Review (PDR) process

- IT infrastructure and applications are moved to a cloud based solution.

- We can demonstrate how risk has been actively managed to support objectives being achieved.

\section{Main risks}

- Capacity and resources to implement change across the different pieces of work that make up this strategic priority

- Revalidation and registrant online services work is being delivered by the same team

- The refreshed values and behaviours will be incorporated into the new way of recruiting which will have a values based focus.

- Objectives will be clearly articulated and success defined at all levels in the organisation

- A member of Senior Leadership Group will act as a risk champion
- Effectiveness of senior decision making

- Interdependencies between multiple pieces of work

- Cynicism/frustration at the pace of change 


\section{Outline timetable:}

\begin{tabular}{|c|c|c|c|}
\hline April-June 2018 & July-September 2018 & October-December 2018 & January-March 2019 \\
\hline $\begin{array}{l}\text { - Revalidation: Launch of myGPhC } \\
\text { during April. Registrants start } \\
\text { recording their revalidation } \\
\text { records } \\
\text { - Revalidation: Second phase of } \\
\text { revalidation development } \\
\text { covering record submission and } \\
\text { exceptional circumstances } \\
\text { - Development, testing and release } \\
\text { of online registration applications } \\
\text { for pharmacists } \\
\text { - Stabilisation of new Azure } \\
\text { infrastructure } \\
\text { - Re-setting the culture: Schedule } \\
\text { workshop sessions with the heads } \\
\text { of function to present the new } \\
\text { culture statement and collate } \\
\text { their feedback } \\
\text { - Re-setting the culture: Agree the } \\
\text { refreshed values with SLG and } \\
\text { communicate to all employees } \\
\text { - Re-setting the culture: Monitor } \\
\text { and evaluate if there has been an } \\
\text { increase / decrease / stabilising of } \\
\text { staff turnover during the period of } \\
\text { implementing the cultural re- } \\
\text { design }\end{array}$ & $\begin{array}{l}\text { - Revalidation: Testing and release of } \\
\text { second phase of revalidation } \\
\text { services. Registrants start to submit } \\
\text { their revalidation records online } \\
\text { from September } \\
\text { - Initial scoping for moving SharePoint } \\
\text { and infrastructure services to the } \\
\text { cloud } \\
\text { - Registrant survey: Analyse results of } \\
\text { survey and prepare draft report } \\
\text { - Re-setting the culture: Carry out } \\
\text { research to establish how the } \\
\text { cultural element of the GPhC } \\
\text { benchmarks against similar sized } \\
\text { organisations or an inter-regulatory } \\
\text { group within the sector } \\
\text { - Risk: Complete internal context } \\
\text { which describes how the } \\
\text { organisation works } \\
\text { - Risk: Develop guidance document to } \\
\text { assist coaching of risk owners } \\
\text { - Risk: Fully develop register of new } \\
\text { strategic risks } \\
\text { - Risk: Provide update on ISO work to } \\
\text { Council }\end{array}$ & $\begin{array}{l}\text { - Revalidation: Further development } \\
\text { of revalidation and registrant online } \\
\text { services } \\
\text { - Finalisation on approach, to move } \\
\text { SharePoint and infrastructure } \\
\text { services to the cloud } \\
\text { - Phased migration of SharePoint and } \\
\text { infrastructure services to the cloud } \\
\text { - Registrant survey: Share and discuss } \\
\text { key findings internally and identify } \\
\text { learnings and actions } \\
\text { - Registrant survey: Approve and } \\
\text { publish report } \\
\text { - Re-setting the culture: Work with } \\
\text { the Learning and Development } \\
\text { Manager to integrate the mapped } \\
\text { culture, values and behaviour } \\
\text { framework into the new PDR } \\
\text { process } \\
\text { - Re-setting the culture: Work with } \\
\text { heads of function to support the } \\
\text { integration of the mapped culture, } \\
\text { values and behaviour information } \\
\text { into a value based recruitment } \\
\text { process }\end{array}$ & $\begin{array}{l}\text { - Testing and release of new online } \\
\text { services for registrants } \\
\text { - Phased migration of SharePoint } \\
\text { and infrastructure services to the } \\
\text { cloud } \\
\text { - Re-setting the culture: March } \\
2019 \text {, carry out the third of } 4 \text { pulse } \\
\text { surveys } \\
\text { - Re-setting the culture: Work with } \\
\text { the Learning and Development } \\
\text { Manager to support the } \\
\text { integration and rollout of the new } \\
\text { behaviour framework into the } \\
\text { new PDR process } \\
\text { - Risk: Develop risk appetite } \\
\text { statement(s) } \\
\text { - Risk: Launch updated framework } \\
\text { - EDI: Develop further guidance } \\
\text { supporting ElAs to ensure that the } \\
\text { 'circle' of impact assessment is } \\
\text { completed }\end{array}$ \\
\hline
\end{tabular}


- Risk: Strategic risks have been refreshed and mapped against current strategic aims to ensure they represent current objectives - Risk: Function and project level risks reviewed and analysed to enhance understanding of the GPhC risk profile

- EDI: Strengthen the GPhC's capability to provide access, adjustments and raise awareness of people with disabilities.
- EDI: Produce a transgender policy to ensure that the GPhC is able to provide access, adjustments and raise awareness of people who are transgender
- Risk: Further develop risks that sit below strategic level

- Risk: draft strategy and other documents for ISO alignment

- EDI: Produce a draft health and wellbeing strategy

- EDI: Support the collection and analysis of GPhC EDI data

\section{Commentary for Q1:}

\section{Improve online services to enable registrants to complete and review their revalidation records online}

As part of the revalidation launch, myGPhC went live in April 2018. Registrants have been signing up to myGPhC and started to record their revalidation entries. The sign-up rate has been higher than expected at 48,200 (as at end of June 2018), and there have been no significant technical issues with myGPhC since the launch. We did experience a higher than expected volume of calls and emails to our Customer Contact Centre as some registrants sought additional assistance in being guided through the sign-up process.

Development is in progress for the next go live in August covering submission of records for review and exceptional circumstances. Work is currently on schedule for this release.

\section{Improve online services for registration, renewal and application functions in phases throughout the year}

The initial phase of the registrant online services covering pharmacist registration applications went live to schedule in June 2018 ahead of the summer registration assessment.

\section{Embed equality, diversity and inclusion in both our role as a regulator and employer}

The EDI Leadership Group review Equality Impact Assessment (EIA) documents and provide feedback to heads of function and managers. To help in the early development process heads of functions have been offered regular support for EIA and other EDI policy development.

EDI raising awareness activities for quarter 1 include:

- A transgender sub-group meeting was set up in June and will take place in September 
- The Disability Network Strategy group's first meeting was held. There was a high level of support and positive feedback from other regulators in establishing a joint network.

\section{Re-setting the culture}

We worked with an external facilitator to deliver two cultural workshops with SLG and as a result the culture statement was agreed while the organisational values were refreshed. The culture statement and refreshed values will be communicated to teams in the form of a 'roadshow'. All team managers were contacted and asked to provide their team's availability for the roadshow presentations.

\section{IT infrastructure}

During April, work has been focused on the post launch support of myGPhC. Work during this period has also included the stabilisation of the new Azure infrastructure.

\section{Business Planning}

We are continuing to progress with developing a new financial strategy, reviewing our wider regulatory strategy and approach, including making sure this is more grounded in operational reality, and applying the insights from the internal audit reviews on transformation and cost metrics.

\section{Risk Management}

Alignment to the ISO risk management standard takes elements of what the organisation has been doing in managing risk, refocuses it and applies the result to an environment in which there is greater joined-up understanding of the work we are delivering as an organisation. We are in the design, discovery and mapping phase. Work to describe the internal context at function level has been completed, and we are now mapping this to the governed decision-making structures. Strategic risks have been refreshed and, to validate consistency with ISO 31000 definitions, mapped again priorities articulated in the 2018/19 Annual Plan. Coaching and support has also been provided to risk owners to assist the further development of function-level risks.

The overall RAG status remains amber although there has been good delivery against actions. Following approval of the revised budget for the $2018-19$ financial year, activities will continue to be reviewed and prioritised. 
General

Pharmaceutical

Council

\section{Meeting paper}

Council on Thursday, 13 September 2018

\section{Public business}

\section{Reporting on the June 2018 Registration Assessment}

\section{Purpose}

To update Council on candidate performance in the June 2018 Registration Assessment.

\section{Recommendations}

Council is asked to note:

i. candidate performance data (Appendix 1) and our response to feedback on the June 2018 Registration Assessment from the British Pharmaceutical Students' Association (BPSA); and

ii. the Board of Assessor's report to Council and associated documents (Appendices 2, 2A and 2B) and the assurance they provide about the June 2018 sitting.

\section{Introduction}

1.1. Passing the GPhC's Registration Assessment is a pre-requisite for applying to register as a pharmacist. There are two sittings every year, in June and September. This paper discusses the June 2018 sitting.

\section{Summary}

2.1. 2942 candidates sat our registration assessment on 28 June 2018, with 2318 candidates passing the assessment; a pass rate of $\mathbf{7 8 . 8 \%}$. There were 2643 candidates who sat the assessment for the first time; 145 for the second time and 154 for the third time. Pre-registration trainee pharmacists are permitted three attempts at the registration assessment. This year's pass rate 
of $78.8 \%$ compares with the $78.2 \%$ pass rate for the June 2017 registration assessment. Further details of candidate performance are set out in Appendix 1.

\section{The BPSA's report}

3.1. Each year the BPSA collects feedback from candidates who sat the Registration Assessment. The feedback is collated in to a report which we and the Board of Assessors consider. In its report on the June 2018, the BPSA made 20 recommendations. Five of these relate to the operation of the Assessment and our responses are set out below. The other 15 concern the content of the Assessment and are addressed by the Board of Assessors in Appendix 2B.

3.2. Recommendation 1: The GPhC should ensure that there is adequate temperature control at the venues that they select for the Registration Assessment, particularly in June. Pharmacy associations like the RPS, BPSA, etc. should work with the GPhC to advise candidates on how to self-manage their own temperature; for example, wear light clothing at high temperatures: The weather on the day of the June 2018 sitting was particularly hot, so as a precautionary measure we emailed all candidates the day before, advising them to bring bottled water and to dress appropriately. All the June 2018 venues except two were air-conditioned: we will contact the two that are not air conditioned in advance of next year's sitting to discuss what arrangements they might make to regulate the temperature, should it be likely that the weather will be hot again.

3.3. Recommendation 2: The GPhC should ensure more specific details are provided where the assessment places might be difficult to find: We will consider whether more can be done to provide additional information about venues for candidates, although we do send web links to venues to candidates already and they do include location and transportation information. This information is on our website as well.

3.4. Recommendation 3: The GPhC should publish the specification of the desks used in the preassessment briefs that students receive (or on their website) with some advice on how to manage the desk space provided if larger desks cannot be provided: Desks are $600 \mathrm{~mm} \times 600 \mathrm{~mm}$ - this information will be added to the Registration Assessment Specification on our website. Regarding the second point, we believe it is the responsibility of candidates to manage desk space appropriately.

3.5. Recommendations 4 \& 5: These are dealt with in the Board of Assessors' report on the sitting.

3.6. Recommendation 6: The GPhC should publish in the Board of Assessors' report the proportion of questions from high, medium and low weight, from the registration assessment framework: This is always provided: see the Board's report accompanying this paper sitting for details. 
3.7. Recommendation 7: This is dealt with in the Board of Assessors' report on the sitting.

3.8. Recommendation 8: To give candidates a better understanding, the GPhC should produce a document detailing the process followed to produce the Registration Assessment: Since introducing our new style examination in 2016, on our website there have been YouTube videos about the Registration Assessment, including how it is produced (https://www.pharmacyregulation.org/preregmanual), and flow diagrams explaining how papers are developed and marked (section 5.3 of https://www.pharmacyregulation.org/theregistration-assessment).

3.9. Recommendations 9-20: These are dealt with in the Board of Assessors' report on the sitting.

\section{Other matters}

4.1. Data releases: All candidate performance data releases comply with the EU's General Data Protection Regulation (2016, implemented 2018) and Freedom of Information Act 2000. As a general principle we release as much data as possible while ensuring that what is presented preserves the anonymity of individuals. This means that some data are not reported. As well as the data presented in this paper, in several weeks there will be a further data release of (1) performance by pre-registration training provider and (2) an anonymised list of candidate results.

4.2. Data reporting cut-offs: In a previous Council meeting we were asked to consider the rationale for using different cut-off points for data reporting (cut-offs being the minimum number of data items in a category used for public reporting). Our practice has been to use our knowledge of data categories and of others with which they might be combined to derive a cut-off point. This has resulted in different points between categories. We have consulted the external statisticians who provide us with Registration Assessment performance data and their advice was that agreeing cut-offs is a balance between professional judgement based on one's knowledge of the data sets in question, transparency in reporting, the validity of reporting and protecting the confidentiality of data subjects, rather than being an exact science. Were we to apply a uniform cut off for all data categories it might disallow us from reporting some data categories in some circumstances, so we plan to continue to apply different cut offs based on our knowledge of the data sets. We will however endeavour to make the cut offs as consistent as possible. Members are asked to note that it is routine for us to report on fewer categories for September sittings because the data sets are smaller. 


\section{Equality and diversity implications}

5.1. As is the case for every sitting of the Registration Assessment, we considered a range of adjustment requests for the June 2018 sitting. The data are as follows:

- 207 adjustment requests granted (183 in June 2017);

- 21 requests granted partially (23 in June 2017). When a request is granted partially it is because the request and supporting evidence does not justify granting the request in full; and

- 7 requests not granted ( 2 in June 2017). Requests are not granted when the request and supporting evidence does not justify granting the request.

All assessment centres can accommodate adjustment candidates.

\section{Communications}

6.1. This paper is a public document but it will be shared directly with relevant stakeholders.

\section{Resource implications}

7.1. There are no current resource implications for the GPhC.

\section{Risk implications}

8.1. There are no risks associated with this report.

\section{Monitoring and review}

9.1. The Registration Assessment is reviewed after every sitting by the GPhC and Board of Assessors. The Board reports on each sitting to Council and the chair attends Council once a year to discuss the year's sittings.

\section{Recommendations}

Council is asked to note: 
i. candidate performance data (Appendix 1) and our response to feedback on the June 2018 Registration Assessment from the British Pharmaceutical Students' Association (BPSA); and

ii. the Board of Assessor's report to Council and associated documents (Appendices 2, 2A and 2B) and the assurance they provide about the June 2018 sitting.

\section{Damian Day, Head of Education}

General Pharmaceutical Council

damian.day@pharmacyregulation.org

6 September 2018 


\section{June 2018 Registration Assessment performance breakdown by characteristic}

Table 1a: Overall performance

\begin{tabular}{|c|c|c|c|c|c|c|}
\hline \multirow[b]{3}{*}{$\begin{array}{l}\text { Number of } \\
\text { candidates }\end{array}$} & \multirow[b]{3}{*}{$\begin{array}{l}\text { Number of } \\
\text { passing } \\
\text { candidates }\end{array}$} & \multirow[b]{3}{*}{$\%$ pass rate } & \multicolumn{2}{|c|}{ Part 1} & \multicolumn{2}{|c|}{ Part 2} \\
\hline & & & \multirow{2}{*}{$\begin{array}{c}\text { Total number } \\
\text { of marks } \\
\text { available }\end{array}$} & \multirow{2}{*}{$\begin{array}{c}\text { Average } \% \\
\text { mark }\end{array}$} & \multirow{2}{*}{$\begin{array}{c}\text { Total number } \\
\text { of marks } \\
\text { available }\end{array}$} & \multirow[b]{2}{*}{$\begin{array}{c}\text { Average \% } \\
\text { mark }\end{array}$} \\
\hline & & & & & & \\
\hline 2942 & 2318 & 78.8 & $39 *$ & 78.0 & $117^{*}$ & 75.9 \\
\hline
\end{tabular}

*In a sitting, there are 40 questions in Part 1 and 120 questions in Part 2. At the post-assessment stage, the Board of Assessors may remove a question or accept more than one answer for a question, if there is evidence to support doing so. In this sitting, the Board of Assessors removed one question from Part 1, accepted two answers for one question in Part 1 and removed three questions from Part 2. This adjusted the number of marks available to 39 in Part 1 and 117 in Part 2.

Table 1b: Paper pass marks

\begin{tabular}{|l|l|}
\hline Paper & 24 (out of 39$)$ \\
\hline Part 1 & 78 (out of 117) \\
\hline Part 2 & $\begin{array}{l}\text { To pass the Registration Assessment, both parts must be passed. } \\
\text { The number of questions required to pass each part may vary from paper to paper and year to year depending } \\
\text { on the difficulty of questions and papers. }\end{array}$ \\
$\begin{array}{l}\text { Note that the number of questions required to pass is the standard and the pass rate is the percentage of } \\
\text { candidates who met the standard. }\end{array}$
\end{tabular}


Table 2: Performance by sitting attempt

\begin{tabular}{|cccc|}
\hline $\begin{array}{c}\text { Sitting } \\
\text { attempt }\end{array}$ & $\begin{array}{c}\text { Number of } \\
\text { candidates }\end{array}$ & $\begin{array}{c}\text { Number of } \\
\text { passing } \\
\text { candidates }\end{array}$ & $\begin{array}{c}\text { \% pass } \\
\text { rate }\end{array}$ \\
\hline 1st & 2643 & 2150 & 81.3 \\
\hline 2nd & 145 & 81 & 55.9 \\
\hline 3rd & 154 & 87 & 56.5 \\
\hline
\end{tabular}

Note that data in Table 3 onwards are for $1^{\text {st }}$ attempt sitters not the full cohort

Table 3: $1^{\text {st }}$ attempt by education route

\begin{tabular}{|ccc|c|c|}
\cline { 3 - 4 } \multicolumn{1}{c}{} & \multicolumn{2}{c|}{ Average \% mark } \\
\hline Education route*** & $\begin{array}{l}\text { Number of } \\
\text { candidates }\end{array}$ & \% pass rate & Part 1 & Part 2 \\
\hline OSPAP & 54 & 79.6 & 75.6 & 78.4 \\
\hline MPharm & 2588 & 81.4 & 79.2 & 76.7 \\
\hline
\end{tabular}

*** One EEA candidate has not been reported.

Table 4: $1^{\text {st }}$ attempt by sex

\begin{tabular}{|ccc|c|c|}
\cline { 3 - 4 } \multicolumn{1}{c|}{} & \multicolumn{2}{c|}{ Average \% mark } \\
\hline Sex & $\begin{array}{c}\text { Number of } \\
\text { candidates }\end{array}$ & \% pass rate & Part 1 & Part 2 \\
\hline Male & 946 & 81.7 & 80.1 & 76.3 \\
\hline Female & 1697 & 81.1 & 78.5 & 76.9 \\
\hline
\end{tabular}

Table 5: $1^{\text {st }}$ attempt by age range

\begin{tabular}{|ccc|c|c|}
\cline { 3 - 5 } & & \multicolumn{2}{c|}{ Average \% mark } \\
\hline Age Range & $\begin{array}{c}\text { Number of } \\
\text { candidates }\end{array}$ & \% pass rate & Part 1 & Part 2 \\
\hline 36 and over & 63 & 36.5 & 60.0 & 75.1 \\
\hline $26-35$ & 279 & 61.3 & 70.3 & 72.3 \\
\hline 25 and under & 2301 & 85.0 & 80.6 & 77.6 \\
\hline
\end{tabular}


Table 6: $1^{\text {st }}$ attempt by country of training

\begin{tabular}{|ccc|c|c|}
\cline { 3 - 4 } \multicolumn{1}{c|}{} & \multicolumn{2}{c|}{ Average \% mark } \\
\hline Country & $\begin{array}{c}\text { Number of } \\
\text { candidates }\end{array}$ & \% pass rate & Part 1 & Part 2 \\
\hline Wales & 94 & 87.2 & 81.6 & 79.3 \\
\hline Scotland & 173 & 85.0 & 81.7 & 77.7 \\
\hline England & 2376 & 80.9 & 78.8 & 76.5 \\
\hline
\end{tabular}

Table 7: $1^{\text {st }}$ attempt by sector

\begin{tabular}{|ccc|c|c|}
\cline { 3 - 5 } \multicolumn{1}{c|}{} & \multicolumn{2}{c|}{ Average \% mark } \\
\hline Sector********* & $\begin{array}{c}\text { Number of } \\
\text { candidates }\end{array}$ & \% pass rate & Part 1 & Part 2 \\
\hline Hospital & 759 & $95.7 \%$ & 85.2 & 72.4 \\
\hline Community & 1863 & $75.5 \%$ & 76.5 & 74.3 \\
\hline
\end{tabular}

$* * * *$ A candidate's sector refers to the placement of the longest duration. If placements of equal duration were undertaken the sector of the most recent placement has been used.

$* * * * *$ Numbers of candidates from academia, GP practices, industry and prisons are too low to report $(<20)$.

Table 8: $1^{\text {st }}$ attempt by ethnicity ( $\geq 20$ candidates in a category)

\begin{tabular}{|ccc|c|c|}
\cline { 3 - 4 } \multicolumn{1}{c|}{} & \multicolumn{2}{c|}{ Average \% mark } \\
\hline Ethnicity & $\begin{array}{c}\text { Number of } \\
\text { candidates }\end{array}$ & \% pass rate & Part 1 & Part 2 \\
\hline Asian - Other & 177 & 73.4 & 76.2 & 74.4 \\
\hline Bangladeshi & 81 & 74.1 & 76.5 & 72.6 \\
\hline Black - African & 219 & 66.2 & 70.6 & 78.0 \\
\hline $\begin{array}{c}\text { Chinese/ } \\
\text { Chinese British }\end{array}$ & 229 & 89.5 & 78.8 & 76.3 \\
\hline Indian & 466 & 82.2 & 77.1 & 75.3 \\
\hline Other ethnic group & 83 & 74.7 & 72.8 & 72.7 \\
\hline Pakistani & 408 & 71.6 & 85.2 & 81.1 \\
\hline White - British & 679 & 92.5 & 80.3 & 76.1 \\
\hline White and Asian & 24 & 83.3 & 77.9 & 77.4 \\
\hline White- Other & 73 & 79.5 & & \\
\hline
\end{tabular}

The following categories have not been reported because they contain <20 candidates: Black-Caribbean, Black-Other, Other Mixed, White-Irish, White and Black African, White and Black Caribbean. In addition, 'Not supplied' and 'NULL' have not been reported. 
Table 9: $1^{\text {st }}$ attempt by school of pharmacy attended (MPharm degree)

\begin{tabular}{|c|c|c|c|c|}
\hline \multirow[b]{2}{*}{ School of Pharmacy $* * * * * *$} & \multirow[b]{2}{*}{$\begin{array}{l}\text { Number of } \\
\text { candidates }\end{array}$} & \multirow[b]{2}{*}{$\%$ pass rate } & \multicolumn{2}{|c|}{ Average \% mark } \\
\hline & & & Part 1 & Part 2 \\
\hline Aston University & 118 & 89.8 & 81.1 & 77.8 \\
\hline University of Bath & 103 & 93.2 & 85.8 & 81.8 \\
\hline University of Birmingham & 55 & 83.6 & 79.6 & 77.0 \\
\hline $\begin{array}{c}\text { University of Bradford } \\
\text { (4-year continuous degree) }\end{array}$ & 69 & 73.9 & 73.2 & 73.4 \\
\hline $\begin{array}{c}\text { University of Bradford } \\
\text { (5-year sandwich degree) }\end{array}$ & 75 & 78.7 & 75.6 & 77.0 \\
\hline University of Brighton & 64 & 70.3 & 75.3 & 73.3 \\
\hline Cardiff University & 103 & 92.2 & 85.0 & 81.5 \\
\hline University of Central Lancashire & 95 & 56.8 & 68.9 & 69.2 \\
\hline De Montfort University & 113 & 75.2 & 75.0 & 72.8 \\
\hline University of Durham & 29 & 82.8 & 77.2 & 78.7 \\
\hline University of East Anglia & 96 & 86.5 & 83.8 & 78.6 \\
\hline University of Hertfordshire & 78 & 73.1 & 73.4 & 73.7 \\
\hline University of Huddersfield & 68 & 77.9 & 78.8 & 76.3 \\
\hline Keele University & 70 & 75.7 & 79.5 & 75.6 \\
\hline King's College London & 88 & 90.9 & 83.0 & 78.0 \\
\hline Kingston University & 101 & 65.4 & 70.4 & 71.4 \\
\hline Liverpool John Moores University & 130 & 78.5 & 79.3 & 76.6 \\
\hline University of Manchester & 102 & 85.3 & 80.3 & 78.5 \\
\hline $\begin{array}{c}\text { Medway School of Pharmacy } \\
\text { (universities of Greenwich and Kent) }\end{array}$ & 98 & 87.8 & 79.3 & 76.9 \\
\hline University of Nottingham & 160 & 90.6 & 85.3 & 79.7 \\
\hline
\end{tabular}




\begin{tabular}{|ccc|c|c|}
\hline University of Portsmouth & 104 & 72.1 & 74.9 & 77.0 \\
\hline University of Reading & 101 & 74.3 & 79.6 & 74.2 \\
\hline The Robert Gordon University & 89 & 76.4 & 82.6 & 78.6 \\
\hline University of Strathclyde & 133 & 90.2 & 80.8 & 82.5 \\
\hline University of Sunderland & 101 & 89.1 & 84.8 & 70.5 \\
\hline University College London & 140 & 93.6 & 65.7 & 69.9 \\
\hline
\end{tabular}

******Data have not been presented (1) for OSPAP providers, (2) for The Queen's University, Belfast and University of Ulster (most of whose graduates sit the PSNI's Registration Examination in Northern Ireland) and (3) the five-year integrated degrees at the University of East Anglia and University of Nottingham, because in all cases candidate numbers are $<20$. 


\section{Report to the Council of the General Pharmaceutical Council on the June 2018 Registration Assessment}

\section{Introduction}

1.1 The initial education and training of pharmacists in Great Britain is:

- a four-year MPharm degree accredited by the $\mathrm{GPhC}^{1}$; then

- 52 weeks of pharmacist pre-registration training; and

- the GPhC's Registration Assessment.

1.2 During pre-registration training, trainees are signed-off on four occasions by a designated pharmacist tutor - at 13, 26, 39 and 52 weeks. Trainees must have been signed off as 'satisfactory' or better at 39 weeks to be eligible to enter for a sitting of the Registration Assessment.

1.3 The Registration Assessment is an examination with two papers: part 1 (morning) and part 2 (afternoon). It is set and moderated by the Board of Assessors, the authors of this report.

1.4 Part 1: The part 1 paper is two hours long (120 minutes) and comprises 40 calculations questions. Calculators are permitted in Part 1.

1.5 Part 2: The part 2 paper is two and a half hours long (150 minutes) and comprises 120 questions: 90 are single best answer questions (SBAs) and 30 are extended matching questions (EMQs). Calculators are not permitted in Part 2.

1.6 Resource packs are provided for candidates, one for each part, and candidates are not permitted to bring any reference sources to the sitting. Examples of resources provided include extracts from

\footnotetext{
${ }^{1}$ Non-EEA pharmacists wanting to register in GB take a one-year university Overseas Pharmacists' Assessment Programme (OSPAP) instead of an MPharm degree.
} 
reference sources such as the national formularies (BNF \& C-BNF), summaries of product characteristics (SPCs) as well as photographs, charts and tables.

1.7 Candidates with a specific need may ask for an adjustment to be made in the conduct of the assessment.

1.8 Candidates with specific needs may sit the assessment in a separate adjustments room and all centres have adjustment rooms.

\section{Reporting to Council}

2.1 There are two sittings of the Registration Assessment every year, in June and September, and the Board of Assessors reports to the GPhC's Council after each one. This is the report for June 2018.

\section{June 2018 summary statistics}

\begin{tabular}{|l|l|l|}
\hline 1. Candidate numbers & Number & $\%$ of total \\
\hline & & \\
\hline Total number of candidates & 2942 & 100.0 \\
\hline & & \\
\hline Number of first sitting candidates & 2643 & 89.8 \\
\hline Number of second sitting candidates & 145 & 4.9 \\
\hline Number of third sitting candidates & 154 & 5.2 \\
\hline
\end{tabular}

\begin{tabular}{|l|l|l|}
\hline Candidate performance - pass rates & Number & $\%$ \\
\hline & & \\
\hline Overall pass & 2318 & 78.8 \\
\hline Overall fail & 624 & 21.2 \\
\hline & & \\
\hline First sitting candidates - pass & 2150 & 81.3 \\
\hline Second sitting candidates - pass & 81 & 55.9 \\
\hline Third sitting candidates - pass & 87 & 56.5 \\
\hline
\end{tabular}




\section{Paper and question analysis}

\section{Question performance}

4.1 The questions performed as expected in both papers. One question removed from Part 1 and two answers were accepted for another question. Three questions were removed from Part 2 . This means that the pass mark for Part 1 was calculated using 39 questions not 40 and the pass mark for Part 2 was calculated using 117 questions not 120.

\section{The balance of questions}

4.2 The balance of questions was consistent with the requirements of the Registration Assessment Framework (see https://www.pharmacyregulation.org/53-registration-assessment-framework):

Total \% of the paper questions high-weighted outcomes: $66.0 \%$

Total $\%$ of the paper questions medium-weighted outcomes: $27.1 \%$

Total \% of the paper questions low-weighted outcomes: $6.9 \%$

\section{Standard setting}

5.1 Setting the standard: the standard of each question is set by a panel of standard setters who are all pharmacists with current experience of pre-registration trainees and early-years pharmacists. The standard of a paper is set based on the standard set for each question. Further information on creating papers and setting standards can be found at https://www.pharmacyregulation.org/education/pharmacist-pre-registration-training-scheme/keydates-scheme/registration-assessment.

5.2 Pass requirements: In order to pass the Registration Assessment, both Part 1 and Part 2 must be passed. The number of questions required to pass each part in this sitting are:

Part 1: 24 questions (/39 questions)

Part 2: 78 questions (/117 questions) 


\section{Feedback to candidates}

6.1 Feedback to candidates is attached as Appendix 2B (and has been available on our website since late August).

\section{Feedback to the BPSA}

7.1 As in previous years, the Board would like to thank the BPSA for its report, which was considered at the Board's meeting on the $19^{\text {th }}$ July 2018 . The report was based on 266 responses, $9 \%$ of the candidates who sat the Registration Assessment in June 2018.

7.2 Some of the recommendations made by the BPSA are operational and will be considered by the GPhC and some are out of scope for the Board, such as securing study time for trainees and training tutors.

7.3 The remaining points have been discussed in the Board's response to the BPSA in Appendix 2B.

Professor Andy Husband, Chair, on behalf of the

Board of Assessors

$5^{\text {th }}$ September 2018 


\section{Feedback on the June 2018 Registration Assessment}

\section{About this document}

This document contains feedback drawn from candidate performance in the General Pharmaceutical Council's (GPhC's) June 2018 Registration Assessment. It is from the Board of Assessors, the body that sets and moderates the Registration Assessment. It is aimed at pre-registration trainee pharmacists, pre-registration tutors and anyone else involved in pharmacist education and training.

\section{The Registration Assessment Framework}

All questions in the Registration Assessment are derived from the Registration Assessment Framework. The different outcomes have different weighting and candidates should use the Framework as the basis for preparation. Many questions in the Assessment will concern patients who have co-morbidities and these questions are mapped across multiple parts of the framework.

\section{Part 1}

Candidates performed well in questions testing the ability to calculate doses and quantities to supply.

Candidates performed less well in questions that related to:

- those involving the Part 1 resource pack, as some candidates omitted these questions; and

- those requiring rounding within the calculation.

Normal mathematical rules about rounding apply to the Registration Assessment. Candidates must read each question carefully and consider for each question where any rounding should occur. In some questions, the rounding should occur at the end of the calculation, but in other questions rounding should occur earlier in the calculation. For example, when calculating the total amount of a medicine that should be supplied, rounding should occur for an individual dose before calculating a final amount. If a question asks for the number of vials required to provide a dose and the candidate calculates 3.2, then 4 vials are needed to provide the required dose. In some questions, specific rounding instructions are provided. For example, a dose may be rounded to the nearest $\mathrm{mL}$ for ease of administration or specific instructions may be given to round up or round down. 
Candidates should check that each answer makes sense.

\section{Part 2}

Candidates performed well in questions testing:

- extraction of information on the frequency of side effects from the provided SPCs;

- when to report an ADR using a Yellow Card except for questions about well-established but serious side effects; and

- the treatment of hypertension.

Candidates performed less well in questions testing decision making when a patient presents with symptoms in a pharmacy. Candidates are expected to know when evidence-based treatment can be recommended and when patients should be referred.

\section{Answer Sheets}

Whilst most candidates filled in their answer sheets as directed, some candidates did not fill in the answer sheets clearly. Marks cannot be awarded if the answer is not clear. Candidates should ensure that numbers in Part 1 are clearly written and that answers in Part 2 are clearly marked on the answer sheet.

In these examples, from June 2018 , the intended answer is not clear.
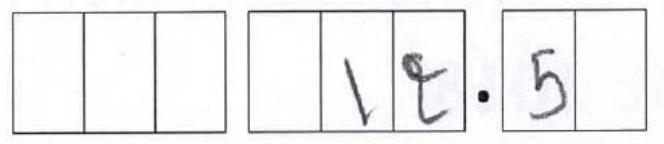
$\% \mathrm{v} / \mathrm{v}$
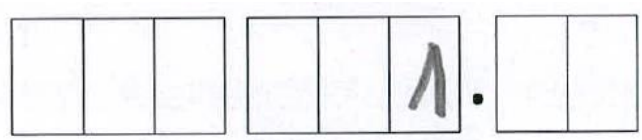

$\mathrm{mL}$

Numbers must be entered in the correct place using the pre-entered decimal point. For example, if the answer is 1292, the following is not correct and the candidate will not be awarded a mark:

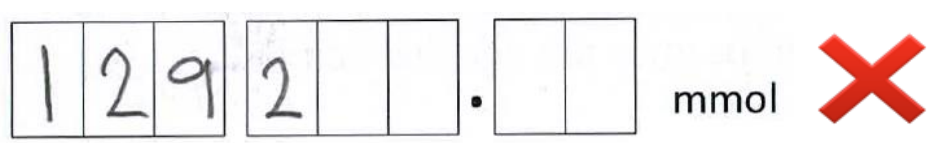


The feedback from previous sittings is available at:

https://www.pharmacyregulation.org/51-registration-assessment-preparation

\section{Professor Andy Husband, chair}

on behalf of the Board of Assessors

$5^{\text {th }}$ September 2018 


\section{Board of Assessors' response to the BPSA's report on the June 2018 Registration Assessment}

The Board of Assessors would like to thank the BPSA for its report ${ }^{1}$ on the June 2018 Registration Assessment. Answers are provided here where the recommendations are within the scope of the Board's work, if they are not, they have been referred to the GPhC.

Recommendation 1: The GPhC should ensure that there is adequate temperature control at the venues that they select for the registration assessment, particularly in June. Pharmacy associations like the RPS, BPSA, etc. should work with the GPhC to advise candidates on how to self-manage their own temperature; for example, wear light clothing at high temperatures: This is dealt with in the GPhC's report on the sitting.

Recommendation 2: The GPhC should ensure more specific details are provided where the assessment places might be difficult to find: This is dealt with in the GPhC's report on the sitting.

Recommendation 3: The GPhC should publish the specification of the desks used in the pre-assessment briefs that students receive (or on their website) with some advice on how to manage the desk space provided if larger desks cannot be provided: This is dealt with in the GPhC's report on the sitting.

Recommendation 4: The BPSA recommends that the GPhC should ensure the resources published and made available to candidates to prepare for the registration assessment are better aligned to the calibre of the actual paper: The resources the Board provides are learning points from previous sittings, the Registration Assessment Framework (including the syllabus) and sample papers. They are all available at https://www.pharmacyregulation.org/51-registration-assessment-preparation. The learning points are added every year and the Framework is refreshed every year to ensure it reflects contemporary practice. The sample questions and questions for live papers are written by the same people and many of the sample questions have been used previously in live papers. For these reasons the Board is confident that the alignment between resources and live papers is good.

\footnotetext{
${ }^{1}$ http://www.bpsa.co.uk/static/pdf/540d328ce2551499791d3b19ccfa98f8.pdf
} 
Recommendation 5: The BPSA recommends that the GPhC ensures an adequate distribution of questions that equate to an average of 3 minutes per question, to allow candidates sufficient time to complete all questions: The amount of time taken to answer a question varies, depending on its length and difficulty. Some require very little time to answer, others require longer. The Board does not have any evidence that there was insufficient time for candidates to complete the papers.

Recommendation 6: The GPhC should publish in the board of assessors report the proportions of questions from high, medium and low weight, from the registration assessment framework: This is dealt with in the GPhC's report on the sitting.

Recommendation 7: The GPhC should ensure that the assessment is an accurate reflection of day-to-day practice to that of a newly qualified pharmacist: Part of the quality assurance of the Registration Assessment is standard setting by the Standard Setters. The Standard Setters are all practising pharmacists with day to day experience of pre-registration trainees and/or recently-registered pharmacists. It is their collective experience that ensures the Registration Assessment is calibrated to the reasonable expectations of a newly-qualified pharmacist.

Recommendation 8: To give candidates a better understanding, the GPhC should produce a document detailing the process followed to produce the registration assessment: This is dealt with in the GPhC's report on the sitting.

Recommendation 9: The GPhC should review and remove all questions which are unnecessarily ambiguous during the moderation of this assessment. In the future, the GPhC should work to avoid unnecessary ambiguity within questions and ensure there is sufficient information to inform a clear single best answer from the options provided: Through its quality assurance processes, the Board makes every effort to ensure that questions are not ambiguous (while maintaining an appropriate level of challenge for candidates). However, as part of its standard review of a paper, the Board always considers whether any questions should be removed in light of their performance. In June 2018, the Board removed one question from Part 1 and three from Part 2. Having reflected on the Part 1 questions, the Board agreed that two answers could be accepted for one question, which could be interpreted in two ways.

Regarding the second point - the need for a clear single best answer - the Board would point out that having a 'clear' and a 'best' answer for the same question is incompatible. This question type, single best answer, requires one answer to be the 'best' answer and for some others to be credible (therefore not clearly wrong). The task for candidates is to distinguish between optimal and credible. A 'clear' correct answer would not reflect the reality of practice in a pharmacy and the need for pharmacists to make critical judgements. Older style questions required knowledge recall alone (allowing a candidate to demonstrate that they 'know' something) but single best answer questions are more nuanced in that they require candidates to demonstrate they 'know how' to apply knowledge. These more sophisticated questions are used across the healthcare professions to test the application of clinical knowledge. 
Recommendation 10: The GPhC should ensure that the calculations within paper 2 of the assessment use simpler numbers and are answerable within the average time limit for each question in this paper: The Board is careful to ensure that the calculations questions in Part 2 can be answered within the time allocated and without a calculator, as could be the case in contemporary practice.

Recommendation 11: The GPhC should restrict the amount of EMQs that require resources to ensure candidates have sufficient time to complete each question: For a given paper, no more than $25 \%$ require the use of a resource. The Board does not distinguish between the use of resources in single best answer and extended matching questions because the question type does not necessarily determine the amount of time needed to refer to the resource.

Recommendation 12: The GPhC should restrict excessively worded EMQs or provide sample questions of similar length to appropriately prepare candidates for these types of questions: The sample EMQs are the same length as the live ones in papers (and some of the sample EMQs were used previously in live papers). The length of the EMQs is normal for that question type.

Recommendation 13: The GPhC should work together with pharmacy associations, such as the RPS and BPSA, and training providers to ensure that candidates understand that they are being assessed for what they have learnt during their pharmacy degree and not just the Pre-Registration training year: The GPhC is happy to work with pharmacy representative bodies to help candidates understand the coverage of the Registration Assessment. As the Registration Assessment focuses on practice, the majority of the questions relate directly to the candidates' experience of pre-registration training (given its proximity to practice). However, where something learnt during the MPharm degree is directly relevant to practice and is included in the Registration Assessment Framework, it may be included in a paper.

Recommendation 14: The BPSA recommends that the GPhC should provide less questions requiring the use of resources and should provide a smaller resources pack: The Board is not clear why providing fewer resource-based question is necessarily a good idea. It would point out that providing a resource pack rather than requiring candidates to bring resources of their own - BNFs, for example - makes it easier for candidates to find the information they need to answer a question because the information will be in the relevant resource.

Recommendation 15: The GPhC should ensure that the Registration Assessment Framework is not misrepresentative nor misleading and provides trainees with sufficient information in order to structure their learning and revision accordingly: The Board reviews the Framework every year to ensure it is accurate and contemporary and that candidates have sufficient information to prepare for the Registration Assessment. The Board reiterates the view that the best way to prepare for the Registration Assessment is not to cram but to engage with as wide a range of patients and clinical scenarios as possible. 
Recommendation 16: Some respondents have recommended that newly qualified Pharmacists should be allowed to review the questions with an experience pharmacist to ensure the level of difficulty is appropriate to a newly qualified Pharmacist: This is the role of the Standard Setters, as explained in the Board's response to Recommendation 7.

Recommendation 17: The GPhC should publish sample questions of representative difficulty, complexity and length to the registration assessment, and choose these questions from the same pool that the registration assessment questions are chosen from: The Board is always careful to provide representative sets of sample questions, while accepting that not every topic included in a paper can be linked to a sample question. The Board makes no distinction between sample and live questions, both of which are written by the same people. Also, some of the sample questions have been used before in live papers.

Recommendation 18: The GPhC should provide at least 2 sample papers for each paper to help candidates have a better understanding of the wording and types of questions as well as a better feel for the timing of the paper: The current sample questions cover all the types of question in a paper and are worded in the same way. The Board has considered whether sample papers should be made available or not and has concluded that they should not. When sample papers were issued in the past, candidates treated them as if they were exact replicas of real papers and relied on them unduly for revision purposes. Not only that, the longer papers remained in the public domain, the less relevant they became.

Recommendation 19: The GPhC should ensure that Pre-Registration trainees are aware that no mock assessments or courses are endorsed by the GPhC and may not provide an accurate insight into the actual GPhC Registration Assessment: The Board reiterates a point it has made before - that it does not endorse any mock assessments or courses because doing so is beyond its remit. We will ensure this is made clear in the documents we produce to support trainees.

Recommendation 20: The BPSA should work closer with the GPhC to help address some of the issues raised in this report and therefore allow candidates to be more prepared for the registration assessment: The Board is always willing to meet with the BPSA to discuss the Registration Assessment.

Professor Andy Husband, Chair, on behalf of the Board of Assessors

$5^{\text {th }}$ September 2018 


\section{Meeting paper}

Council meeting on Thursday, 13 September 2018

\section{Public business}

\section{Pharmacist independent prescribing}

\section{Purpose}

To provide the Council with an overview of pharmacist independent prescribing and the work planned in this area.

\section{Recommendations}

The Council is asked to note this paper.

\section{About pharmacist independent prescribers}

1.1. Regulations to allow pharmacists to prescribe independently came into effect in 2006. A pharmacist prescriber is a pharmacist who has completed the relevant approved education and training to add an annotation ${ }^{1}$ to their entry in the register ${ }^{2}$.

1.2. Pharmacist independent prescribers (PIPs) can prescribe both within the National Health Service and privately, including primary care (including GP practices), secondary care and in healthcare roles in the armed forces and H M Prisons.

1.3. A PIP may prescribe autonomously for any condition within their clinical competence. This currently excludes three controlled drugs for the treatment of addiction.

1.4. Pharmacists working as independent prescribers is part of a wider change in which pharmacy is playing a more significant part in people-facing, front-line care. Pharmacists are being used more and more to support patients through prescribing. This is helping to make the most effective and cost-effective use of medicines and relieving pressure in critical parts of the healthcare system - particularly in emergency departments and in primary care (for example in GP surgeries, medical centres and care homes).

1.5. National pharmacy strategies vary across Great Britain. But there is a consistent recognition that employing pharmacist independent prescribers across healthcare settings:

\footnotetext{
1 "annotation" means an annotation in the Register;

2 "the Register" means the register established and maintained under article 19 of the Pharmacy Order 2010
} 
- makes the best use of pharmacists' prescribing knowledge and skills; and

- complements the skills of other members of healthcare teams who are being asked to work together in ever-closer ways to create integrated care for people.

1.6. The GPhC commissioned a survey of pharmacy professionals in 2013. Through that work we heard that:

- Three quarters (74\%) of prescribers had prescribed at some point since their annotation and of these, $82 \%$ had prescribed in the last 12 months (61\% of all with a prescriber annotation)

- Prescribers were predominantly working in hospital settings (61\%) and primary care settings (30\%), less so in community settings (11\%). Six per cent worked across multiple settings

1.7. In order to qualify as a pharmacist independent prescriber, pharmacists must complete a GPhC accredited programme. On successful completion of the programme, pharmacists will receive a practice certificate in independent prescribing, making them eligible to apply for annotation on the register.

1.8. On 20 August there were 7143 pharmacists annotated as pharmacist independent prescribers.

1.9. The graph below shows the increasing numbers of pharmacist independent prescribers over the last five years. There has been a 105\% increase between the figure for Quarter 12015 16 (3294) and the figure for Quarter 1 2018-19 (6770).

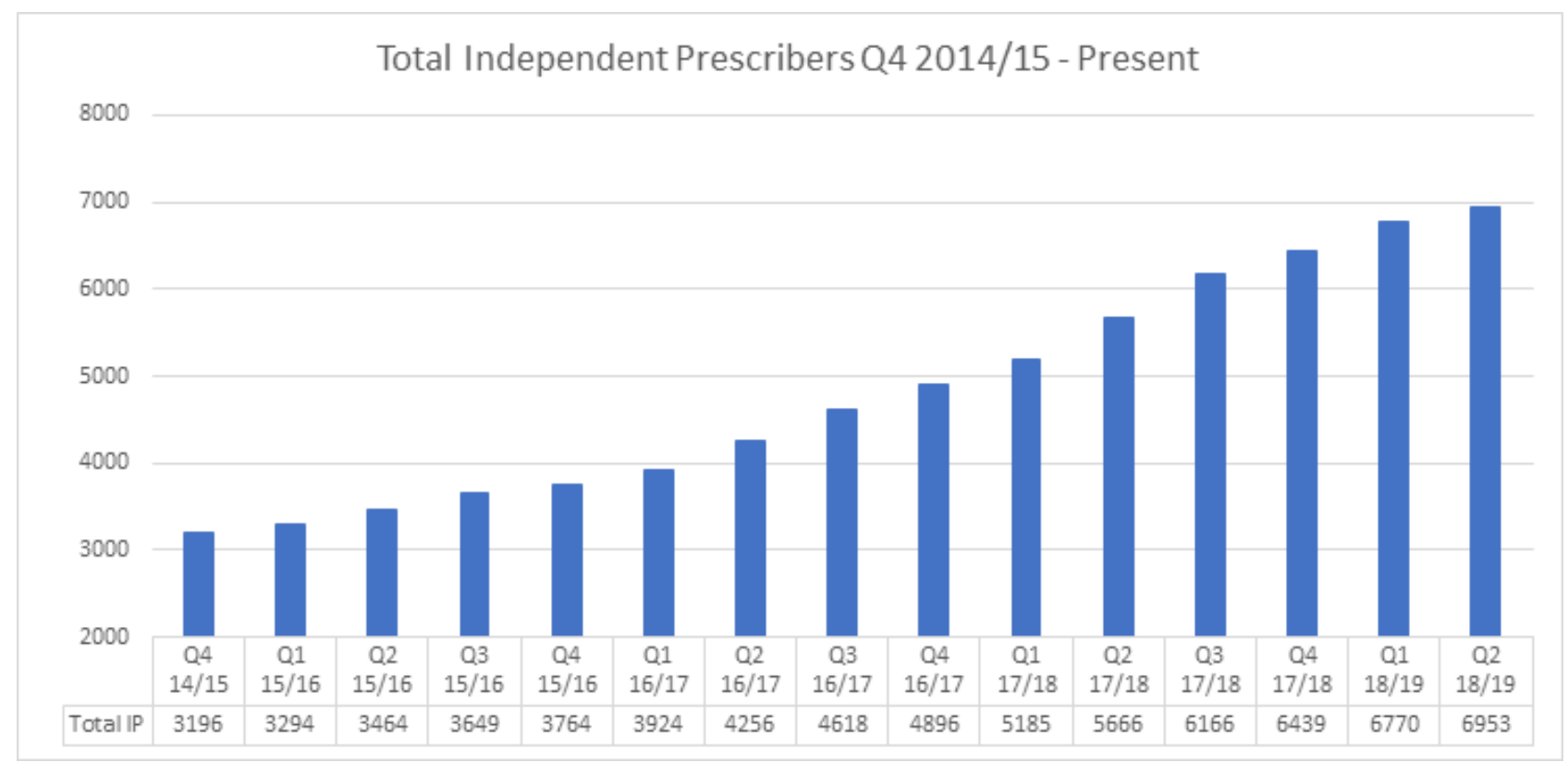


NB: the data for Q2 2018 is not complete and reflects PIP numbers up to 20 August 2018.

\section{Quality assurance of education and training}

\section{a. About IP programmes}

2.1. In order to be annotated as a prescriber a pharmacist must take and pass an education and training course.

2.2. There are 48 IP programmes. Programmes vary depending on the institutions but all must have a pharmacist fully involved in the curriculum development and delivery. 22 of the programmes are nurse-led and are not in a school of pharmacy. 26 are attached to a school of pharmacy.

2.3. Most of the programmes are multi professional, mostly with nurses although a small number also include other healthcare professionals. There are around six programmes that are pharmacist only, which are run by a school of pharmacy. The strength of the multiprofessional programmes is the sharing of knowledge and skills. Pharmacists support the nurses in the pharmacology and numeracy aspects of the course and nurses support pharmacists in developing their clinical skills.

2.4. There are several methods of delivery of the programme. Some offer the programme over a year where the pharmacist has to undertake structured learning activities e.g. attending lectures and practical clinical experience sessions initially and do their learning in practice (90 hours with a designated medical practitioner (DMP)) after the structured learning. Others undertake the learning in practice simultaneously with the learning. Some of the programmes offer a residential course which take place over several weekends or over a week.

2.5. There are two elements to assessing competence on the IP programmes. Pharmacists have to undertake an Objective Structured Clinical Examination which will test a number of aspects which include prescribing, decision making and clinical skills. Pharmacists also have to undertake 90 hours of learning in practice with a designated medical practitioner (DMP). They will cover all aspects of prescribing in a particular area along with clinical and diagnostic skills. At the end of the 90 hours the DMP will sign the following declaration. In my opinion as the DMP, the skills demonstrated in practice confirm the pharmacist as being suitable for annotation as an Independent Prescriber.

2.6. The pharmacist will also submit a portfolio as part of the reflections of this experience to be assessed by the university. The assessment strategy is scrutinised by the accreditation process to ensure pharmacists are fairly assessed and that they demonstrate safe and effective practice. The university quality assurance processes ensure the assessment decisions are externally and independently examined. The DMP declaration is only part of 
the competency assessment, it is never the only element that assesses competence of a pharmacist prescriber.

\section{b. IP programme accreditation}

2.7. The GPhC sets standards for courses leading to annotation as a PIP and accredits them by appointing expert teams to evaluate whether courses meet its standards. The IP programme accreditation cycle is every three years. The criteria for accreditation focus on:

- $\quad$ The course provider: to check whether the provider is capable of delivering a course of the right calibre;

- $\quad$ Entry pre-requisites: to check that providers are evaluating the suitability of applicants to train as prescribers;

- $\quad$ The course: to check that the course will deliver the GPhC's learning outcomes;

- Learning in practice: to check that students have adequate opportunities to practise their diagnostic, consultation and prescribing skills while being supervised by a designated medical practitioner;

- $\quad$ Assessment: to check that students' diagnostic, consultation and prescribing skills (as well as pharmacological knowledge and numeracy) are assessed by appropriate and robust methods; and

- Award: to check that all accreditation criteria have been met and that an academic award can be made.

2.8. When an IP programme is initially accredited it is given provisional accreditation for one year or after the first graduating cohort. A monitoring event takes place at this point to evaluate the experience of the first pharmacist cohort focussing on the clinical skills development. This year (2017/18 academic year) we have had 21 IP events. There have been 15 reaccreditations and 3 accreditations for new IP programmes. There have also been 3 monitoring events for the provisionally accredited IP programmes from last year. There were no conditions imposed on 12 of the IP programmes at their accreditation event but 9 IP programmes had conditions imposed., which meant they did not meet the standards.

2.9. When conditions are imposed, a deadline is set for the condition to be satisfied before accreditation will be granted. Providers must submit evidence to satisfy the condition, which is then evaluated by the accreditation team who will make a recommendation to the registrar. Reports of all accreditations are published on the GPhC website for the full period of accreditation. These reports also include details of the conditions set and how they have been satisfied.

2.10. Two common themes arising from conditions imposed were: 
- Assessment: Providers had to ensure that for assessments concerning patient safety, the regulations were sufficiently stringent to promote safe and effective practice and meet the GPhC learning outcomes. And also that any competency assessments conducted by the DMP were sufficiently quality assured by the university.

- Clinical and diagnostic skills: Providers had to ensure that pharmacists acquire the necessary clinical and diagnostic skills, which is a key outcome for pharmacists and this area comes under particular scrutiny as part of the accreditation process.

\section{Current and emerging work}

\section{a. Regulatory standards and guidance}

3.1 Our primary focus is setting the initial education and training standards for pharmacist independent prescribers and our recent consultation on this is the subject of the accompanying paper to Council.

3.2 The standards for pharmacy professionals apply to all pharmacists and pharmacy technicians, whatever the scope of practice and therefore apply to all pharmacist prescribers.

3.3 The data and information we have clearly indicates that this is an increasing area of practice. In addition, this is a complex area of practice and we know that pharmacist prescribers are working in an increasingly diverse range of settings, including GP practices, care homes and online pharmacies. As a result, we will be producing guidance for pharmacist prescribers to help ensure patients and the public receive safe and effective care.

3.4 In developing this guidance, we will consider guidance that has been produced by other healthcare regulators, for example the General Medical Council, and also the work of professional leadership bodies. We will also consider the feedback we have received through our education and training consultations, for example access to patient records and how patients can be made aware of PIPs, as part of the guidance development. Draft guidance will be subject to consultation and engagement.

\section{b. Working with others}

3.5 We continue to work collaboratively with the Care Quality Commission and other regulators to ensure we are identifying issues and any regulatory gaps. The GPhC is part of a cross regulatory group 'online primary care cross regulatory forum' and have continued to work with the group to explore issues such as the prescribing and dispensing of medicines online. 


\section{Equality and diversity implications}

4.1 There are no equality and diversity Implications associated with this paper.

\section{Communications}

5.1 All of our work to date in relation to prescribing has been informed by views we have heard through ongoing consultation and engagement with our key stakeholders including patients and their representatives, pharmacist prescribers, , pharmacy owners and others. This has included focus groups held with patients and the public, and with pharmacy professionals and pharmacy owners, on what they would expect in relation to the education and training of pharmacist independent prescribers.

5.2 We will continue to proactively communicate and engage with these groups as we take forward the future activities outlined in this paper, including the publication and implementation of new standards for the initial education and training of pharmacist independent prescribers and the development of new guidance for pharmacist prescribers.

\section{Resource implications}

6.1 Individual pieces of work have been resourced within existing budgets.

\section{Risk implications}

7.1 It is important, as prescribing within pharmacy grows, that the GPhC identifies and mitigates any potential risks associated with pharmacist prescribing. The paper outlines the ways in which the GPhC can continue to understand and regulate pharmacist prescribing in way that ensures patients and the public continue to receive safe and effective care from pharmacy professionals.

\section{Monitoring and review}

8.1 The work outlined in the paper will be taken forward and Council will be kept updated on progress as part of ongoing Council business.

\section{Recommendations}

The Council is asked to note this paper. 
Priya Warner, Head of Policy and Standards

General Pharmaceutical Council

priya.warner@pharmacyregulation.org

Tel 02037137958

4 September 2018 


\section{Meeting paper}

Council on Thursday, 13 September 2018

Public business

\section{Scoping research on mechanisms in fitness to practise processes to eliminate discrimination}

\section{Purpose}

To inform the Council on action taken to date and next steps to scope out research on our fitness to practise processes to assure they effectively eliminate discrimination.

\section{Recommendations}

The Council is asked to note the work that has been completed.

The Council is asked to provide comment to inform how work will be carried forward to actively ensure our processes are effective at eliminating discrimination.

The Council is asked to note updated summary data for 2017 for pharmacists and their declared ethnicity in fitness to practice processes compared with the whole population of pharmacists (annex one).

\section{Introduction}

1.1. Our legal duties and ambitions for equality, diversity and inclusion (EDI) are set out in our EDI statement. One of our legal duties is to have due regard to eliminate discrimination in the exercise of our functions.

1.2. Over the last few years we have been undertaking analysis of our data (2017 data provided in annex one) and providing high level reports on fitness to practise and equality and diversity. More recently we have been exploring in more depth how we might research and better analyse how effective our fitness to practise process is at eliminating discrimination. This paper refers throughout to pharmacists; the number of cases involving pharmacy 
technicians is so small that analysis is difficult and the risk of inadvertent identification too high to make it safe to report.

1.3. In our fitness to practise processes, we use a variety of mechanisms to assure good decisionmaking, which includes eliminating discrimination from decisions. Some of these mechanisms include:

1.3.1. Unconscious bias training for staff and key roles in fitness to practise decision-making

1.3.2. Agreed and documented processes for case management which can be quality assured

\subsubsection{Decision-making guidance}

1.3.4. Multiple and joint decision makers both internal and independent from the organisation

\subsubsection{Review of decisions}

1.4. At its meeting in July 2017 the Council agreed the current version of the threshold criteria. At the same time, Council also requested a report on EDI in fitness to practise processes to be brought to them in due course.

1.5. This paper sets out what has been done to scope out the research, provides updated data from 2017 in annex one and presents a recommended plan for further action.

1.6. Research in this area is well known for its limitations because of very small numbers of cases which make meaningful analysis difficult and also risk identification of registrants when published. The purpose of the scoping exercise was to explore those limitations and attempt to find ways to undertake meaningful research activity. These limitations include:

1.6.1. very small numbers of cases which make it difficult to draw meaningful conclusions, or

1.6.2. significant gaps in data because it is provided on a voluntary basis by registrants or people raising concerns.

1.6.3. the complexity of the analysis which draws on quantitative data on case outcomes and also qualitative data about decision-making in cases.

1.6.4. analysis of cases in the fitness to practise process does not acknowledge the wider issues that may affect whether concerns are reported to us (either underor over-reporting).

1.7. It should be noted that the research we are planning is a proactive enquiry to meet our duties to ensure our processes are effective rather than an attempt to remediate a problem we have uncovered. There has been no specific cause to question whether the mechanisms currently being used are effective, but we are actively seeking confirmation of their effectiveness and potential improvements. 
1.8. Additionally, since this work commenced there have been developments in the wider context of health regulation that the Council will wish to consider in their deliberations on how to take the work forward. The proposed timetable that follows in paragraph 3.7 attempts to take into account these developments so they can be factored into further research. These developments include:

1.8.1. the Michalak case and its impact on the handling of claims of discrimination against regulators,

1.8.2. taking a more restorative approach to fitness to practise, which will affect the approach we might use in future to manage concerns,

1.8.3. supporting registrants with their mental health, which would likely be regarded as a protected characteristic in most cases,

1.8.4. the recommendations from the Williams review to hold regulators to a PSA standard related to equality and diversity, to train those involved in fitness to practise processes in equality and diversity, and for PSA to undertake research into the link between legal representation for registrants and fitness to practise outcomes and a further link to protected characteristics.

1.8.5. PSA's recommendations for reform to place patients at the heart of regulation and more specifically fitness to practise processes.

\section{The scoping exercise and its recommendations}

2.1. A scoping exercise was conducted following procurement processes to appoint an independent specialist legal firm.

2.2. The purpose of the scoping exercise was to inform how we might take our next steps to conduct research in this area. As a result, the exercise was inherently small scale and reported limited findings on the data that was analysed. Instead the report provided legal advice on interpretation of our duties under the law, observations on if and how our data could be used and methodological considerations for future research.

2.3. The recommendations below have been made for further work:

2.3.1. Review of the reports commissioned by other regulators to understand if, and how, limitations in data and in meaningful analysis were overcome.

2.3.2. Further quantitative analysis of our data including, if possible, providing it within the context of data from the whole register or national population. It was noted that gaps in data could helpfully be filled to inform that work. For example, information on protected characteristics is not always volunteered from the people raising concerns. 


\subsubsection{An analysis of fitness to practise processes and guidance, in particular for decision points in the process.}

2.4. Following the above steps, recommendations for improvements to our processes and guidance could be formulated and then actioned.

2.5. A recommendation was also made to not conduct further work to analyse specific cases in depth. This was because:

2.5.1. The scoping exercise identified no overt bias from the limited review,

2.5.2. Comparator cases would be difficult to find owing to the wide number of variables that inform decision-making in fitness to practise processes and the low number of cases,

2.5.3. In a high number of instances people involved in cases have not declared if they have protected characteristics which is voluntary.

\section{Recommended plan of activity}

3.1. Using the recommendations from the scoping exercise as a starting point, it is suggested that a more holistic process evaluation is undertaken to fully understand how the different and layered mechanisms used to assure fair decision-making, including eliminating discrimination, are working.

3.2. The timetable sets out an extended period of activity to develop a more fulsome evaluation approach which will afford time to overcome the limitations in data and methodology which have been identified in the scoping exercise.

3.3. The development of a logic model will be an important step to set out the full range of mechanisms that are used to eliminate bias and then test their effectiveness both individually and together.

3.4. Once the logic model has been developed it can be tested by an independent supplier. That testing will look at the model itself to make sure it is comprehensive and rigorous in its logical assertions.

3.5. Following testing of the logic model, it will then be possible to start looking at measures of effectiveness. The methods used to measure effectiveness of our mechanisms to eliminate discrimination will depend on each mechanism. It might be the case that we can conduct that measurement through looking at our own data, or it might mean we need to engage with people affected by our processes to hear their views.

3.6. Once analysed, the data from the evaluation can then be used to make assertions about the effectiveness of our approach, highlight areas for improvement and also areas beyond our control (such as under- or over-reporting of concerns) that we will want to recognise and possibly influence less directly. 
3.7. Based on the report and its recommendations the table below sets out a suggested plan for further action:

\begin{tabular}{|c|c|}
\hline Timetable & Activity \\
\hline September 2018 - December 2018 & $\begin{array}{l}\text { Strategic review of the contextual developments in } \\
\text { regulation related to equality and diversity. }\end{array}$ \\
\hline September 2018 - February 2019 & $\begin{array}{l}\text { Review of other regulator's ongoing or completed } \\
\text { work }\end{array}$ \\
\hline September 2018 - February 2019 & $\begin{array}{l}\text { Develop logic model for the mechanisms that } \\
\text { assure the quality of fitness to practise process } \\
\text { decision-making relevant to eliminating } \\
\text { discrimination }\end{array}$ \\
\hline April 2019 - September 2019 & Improve data collection to aid research \\
\hline April 2019 - September 2019 & Improve data quality to aid research \\
\hline June 2019 - August 2019 & $\begin{array}{l}\text { Tender and commission research to: } \\
\text { - } \quad \text { analyse data and compare to contextual } \\
\text { population data and } \\
\text { - } \quad \text { test the logic model and } \\
\text { - evaluate the effectiveness of the mechanisms } \\
\text { we use to eliminate the potential for } \\
\text { discrimination }\end{array}$ \\
\hline September 2019 - November 2019 & Research activity conducted and report produced \\
\hline December 2019 & $\begin{array}{l}\text { Present report to Council alongside } \\
\text { recommendations for improvements if any are } \\
\text { identified. }\end{array}$ \\
\hline
\end{tabular}

\section{Equality and diversity implications}

4.1. The purpose of the research is to ensure that the mechanisms we use to eliminate discrimination, and the potential for discrimination, from our fitness to practise processes and decision-making are effective. 
4.2. As a result, the implications of this work are to provide confirmation that our ways of working are effective at eliminating discrimination and to seek opportunities to improve. Therefore, the implications should be positive for people with protected characteristics.

4.3. There is also a potential improvement to the quality of data related to volunteered information on protected characteristics from people associated with fitness to practise processes.

\section{Communications}

5.1. At the current time, there is little to be communicated because we do not have any research outcomes to share. On completion of research, the findings will be shared internally and externally. In particular, we will share information with our colleagues at other regulators.

5.2. There is a slight risk that mis-understanding and mis-communication might occur over the fact of our interest in this subject area. As stated earlier in the report, our interest is to provide assurance of effectiveness of our current measures and to seek improvements. However, it may be perceived that this a remedial activity designed to address a problem. Therefore, communications publicly about this subject area will need to be managed.

\section{Resource implications}

6.1. If the Council agrees to the activities and timetable for future work, the resources for this research will be:

\subsection{1. $2018 / 19$}

Staff time to review other regulator's findings and develop the logic model

\subsection{2. $2019 / 20$}

Financial resources would need to be included in budget for an external supplier. Staff time to manage the external supplier would also be required.

\section{Risk implications}

7.1. The work proposed seeks to mitigate still further any possible risk of discrimination in our decision-making. It should be noted that the exercise of conducting the research may highlight areas for improvement which would likely create future work or resource demands in the 2020/21 financial year. For example, costs for supplementary training for staff or partners and associates might be identified.

\section{Monitoring and review}

8.1. The plan will be incorporated into annual business planning following its review by Council. This would mean Council has another opportunity to consider the timetable and work when they approved the annual business plan for $2019 / 20$. If the work is undertaken, monitoring 
of progress would take place through the performance and delivery board. The outcomes from the research would be presented to Council at its meeting in December 2019.

\section{Recommendations}

The Council is asked to note the work that has been completed.

The Council is asked to provide comment to inform how work will be carried forward to actively ensure our processes are effective at eliminating discrimination.

The Council is asked to note summary data from 2017 for pharmacists and their declared ethnicity and sex in fitness to practice processes compared with the whole population of pharmacists (annex one).

\section{Osama Ammar, Head of Revalidation}

General Pharmaceutical Council

osama.ammar@pharmacyregulation.org

Tel 02037137962

Claire Bryce-Smith, Director of Insight, Intelligence \& Inspection

General Pharmaceutical Council

6 September 2019 
1. Updated analysis related to ethnicity and sex and fitness to practise data for pharmacists for 2017 shows:

The ethnicity of our pharmacist registrants by ethnicity (Figure 1).

1.1. The ethnicity of our pharmacist registrants who are referred to fitness practise, where the pharmacist registrant is known (Figure 2).

1.2. Whether there is over representation relating to ethnicity of pharmacist registrants who enter fitness to practise in context of our total population (Figure 3). For example, in 2017, pharmacists in the Not Supplied category (people who did not declare their ethnicity) make up $10 \%$ of the total register. However, people in this Not Supplied category make up $14 \%$ of people who entered the fitness to practise process (concerns raised). This means that people in the Not Supplied category are overrepresented in the fitness to practise process relative to their population size on the register. Specifically, there are $37 \%$ more registrants with Not Supplied ethnicity in the fitness to practise process relative to the total number of registrants that have Not Supplied ethnicity.

1.3. Whether there is over representation relating to sex of pharmacist registrants and concerns raised (Figure 4).

2. The notes on the data provided are:

2.1. All data in Figures 1, 2, 3 and 4 are limited to registered pharmacists only (cases involving pharmacy technicians are extremely small making analysis difficult and the risk of identification significant).

2.2. The register dataset contains all pharmacists that were registered in 2017.

2.3. Where the concern raised had no pharmacist associated, the concern was removed from the dataset.

2.4. The number of concerns relate to a unique pharmacist, if there were more than one concern raised against a pharmacist, the pharmacist has only been counted once.

2.5. No grouping of ethnicity into aggregated groups has been applied to the analysis.

2.6. Ethnicity data is provided on a voluntary basis by registrants so there are gaps in the data, there is a category of "Not supplied" where the ethnicity was not provided.

3. Analysis continues to be challenging owing to the very small data set and its potential to mislead and identify registrants. 
4. Figure 1: Total population by ethnicity of registered pharmacists in 2017

\begin{tabular}{|l|r|}
\hline Ethnicity & \% of Total Register \\
\hline White - British & $37.39 \%$ \\
\hline Indian & $18.04 \%$ \\
\hline Not Supplied & $10.51 \%$ \\
\hline Pakistani & $8.23 \%$ \\
\hline White - Other & $5.70 \%$ \\
\hline Black - African & $5.59 \%$ \\
\hline Chinese / Chinese British & $4.61 \%$ \\
\hline Asian - Other & $3.15 \%$ \\
\hline Other Ethnic Group & $2.17 \%$ \\
\hline White - Irish & $1.74 \%$ \\
\hline Bangladeshi & $1.27 \%$ \\
\hline Other Mixed & $0.46 \%$ \\
\hline White and Asian & $0.43 \%$ \\
\hline Black - Caribbean & $0.29 \%$ \\
\hline White and Black African & $0.18 \%$ \\
\hline Black - Other & $0.16 \%$ \\
\hline White and Black Caribbean & $0.08 \%$ \\
\hline
\end{tabular}


5. Figure 2: Fitness to practise population by ethnicity of concerns raised in 2017

\begin{tabular}{|c|c|}
\hline Ethnicity & $\%$ of Concerns raised \\
\hline Indian & $23.69 \%$ \\
\hline White - British & $23.69 \%$ \\
\hline Pakistani & $12.07 \%$ \\
\hline Not Supplied & $11.44 \%$ \\
\hline Black - African & $8.20 \%$ \\
\hline Asian - Other & $5.59 \%$ \\
\hline White - Other & $4.23 \%$ \\
\hline Not Disclosed & $2.97 \%$ \\
\hline Other Ethnic Group & $2.43 \%$ \\
\hline Chinese / Chinese British & $1.89 \%$ \\
\hline Bangladeshi & $1.26 \%$ \\
\hline White - Irish & $0.99 \%$ \\
\hline Other Mixed & $0.54 \%$ \\
\hline Black - Caribbean & $0.36 \%$ \\
\hline White and Asian & $0.36 \%$ \\
\hline Black - Other & $0.18 \%$ \\
\hline White and Black Caribbean & $0.09 \%$ \\
\hline White and Black African & $0.00 \%$ \\
\hline
\end{tabular}


6. Figure 3: Percentage comparison of ethnicity by population of the register and concerns raised in 2017

\begin{tabular}{|c|c|c|c|c|c|}
\hline Ethnicity & $\begin{array}{l}\text { \% of Total } \\
\text { Register }\end{array}$ & $\begin{array}{l}\% \text { of Concerns } \\
\text { raised }\end{array}$ & $\begin{array}{l}\% \text { Change in } \\
\text { Population }\end{array}$ & $\begin{array}{l}\text { Total number } \\
\text { of registered } \\
\text { Pharmacists }\end{array}$ & $\begin{array}{l}\text { Total number } \\
\text { of concerns } \\
\text { raised }\end{array}$ \\
\hline Asian - Other & $3.15 \%$ & $5.59 \%$ & $7.38 \%$ & 1802 & 62 \\
\hline Black - African & $5.59 \%$ & $8.20 \%$ & $46.66 \%$ & 3199 & 91 \\
\hline Pakistani & $8.23 \%$ & $12.07 \%$ & $46.61 \%$ & 4712 & 134 \\
\hline Not Supplied & $10.51 \%$ & $14.41 \%$ & $\$ 7.16 \%$ & 6014 & 160 \\
\hline Indian & $18.04 \%$ & $23.69 \%$ & $\$ 1.35 \%$ & 10323 & 263 \\
\hline Black - Caribbean & $0.29 \%$ & $0.36 \%$ & $24.98 \%$ & 165 & 4 \\
\hline Other Mixed & $0.46 \%$ & $0.54 \%$ & $77.17 \%$ & $264 \mid$ & 6 \\
\hline Other Ethnic Group & $2.17 \%$ & $2.43 \%$ & $12.08 \%$ & 1242 & 27 \\
\hline Black - Other & $0.16 \%$ & $0.18 \%$ & $9.69 \%$ & 94 & 2 \\
\hline White and Black Caribbean & $0.08 \%$ & $0.09 \%$ & $9.69 \%$ & 47 & 1 \\
\hline Bangladeshi & $1.27 \%$ & $1.26 \%$ & $0.58 \%$ & 726 & 14 \\
\hline White and Asian & $0.43 \%$ & $0.36 \%$ & $-5.83 \%$ & 245 & 4 \\
\hline White - Other & $5.70 \%$ & $4.23 \%$ & $25.72 \%$ & 3262 & 47 \\
\hline White - British & $37.39 \%$ & $23.69 \%$ & $6.63 \%$ & 21395 & 263 \\
\hline White - Irish & $1.74 \%$ & $0.99 \%$ & $4.00 \%$ & 995 & 11 \\
\hline Chinese / Chinese British & $4.61 \%$ & $1.89 \%$ & $\$ 8.93 \%$ & 2636 & 21 \\
\hline White and Black African & $0.18 \%$ & $0.00 \%$ & $100.00 \%$ & 105 & 0 \\
\hline Pharmacist Total & $100.00 \%$ & $100.00 \%$ & & 57226 & 1110 \\
\hline
\end{tabular}

7. Figure 4: Percentage comparison of sex by population of the register and concerns raised in 2017

\begin{tabular}{|c|c|c|c|c|c|}
\hline Sex & $\begin{array}{l}\% \text { of Total } \\
\text { Register }\end{array}$ & $\begin{array}{l}\% \text { of } \\
\text { Concerns } \\
\text { raised }\end{array}$ & $\begin{array}{l}\% \text { Change in } \\
\text { Population }\end{array}$ & $\begin{array}{l}\text { Total number } \\
\text { of registered } \\
\text { pharmacists }\end{array}$ & $\begin{array}{l}\text { Total number } \\
\text { of concerns } \\
\text { raised }\end{array}$ \\
\hline Male & $38.66 \%$ & $64.59 \%$ & $67.09 \%$ & 22123 & 717 \\
\hline Female & $61.34 \%$ & $35.41 \%$ & $-42.28 \%$ & 35103 & 393 \\
\hline Total & & & & 57226 & 1110 \\
\hline
\end{tabular}




\section{Meeting paper}

Council meeting on Thursday, 13 September 2018

\section{Public Business}

\section{Council member appointments 2019}

\section{Purpose}

To consider recommendations on the process for filling Council member vacancies arising in March 2019

\section{Recommendations}

The council is asked to:

I. Note the progress update on the recruitment of Council members through an open competition process

II. Agree the process for filling the remaining vacancies through a reappointments process

\section{Introduction}

1.1. At its meeting in July 2018, Council agreed that five Council member vacancies arising in March 2019 will be filled using an open competition process (these members are not eligible for reappointment). Council also updated the selection criteria and competencies for new Council member appointments and noted the next steps in the appointments process and timetable, including proposals to secure an executive search agency to manage the recruitment process.

\section{Key considerations}

\section{(a) Open competition: a progress update}

2.1. Since the last Council meeting in July, we have secured the services of Saxton Bampfylde to manage the recruitment process. In reviewing proposals, we considered a range of factors and criteria including proven experience in attracting and recruiting diverse and high-calibre candidates; the proposed methodology to complete the recruitment; the constitution and experience of the team; and, the overall cost (to ensure this falls within existing budgets). 
2.2. The selected agency is an established advocate for diversity in appointments across all sectors, and demonstrated that they importance of ensuring a diverse field for senior appointments. They were also able to demonstrate a depth of experience in healthcare and regulation as well as broader cross-sector work across public, private and non-profit sectors, combined with a well-resourced search approach. In particular, examples of recent experience included working with the Professional Standards Authority on the appointment of members to their non-executive board as well as Chair and members appointments within other regulators.

2.3. The PSA has now completed its scrutiny of our 'Advance Notice of Intent to Recommend Appointment' bundle and confirmed that, overall, they are content with the proposed approach. This includes our advertising and communications plans, our approach to recruitment, updated selection criteria and competencies, and our equality and diversity strategy. However, two specific issues were raised:

(a) The PSA have requested that we use an alternative independent panel member (IPM) for this year's selection panel. While there is no specific limit on the number of times that an IPM may be used, the PSA guidelines provide that if a regulator proposes to use an IPM who has served previously, it should assess whether the extent of previous involvement is sufficiently great that the individual would no longer be considered independent of the regulator. We accept this recommendation and are now in the process of appointing an alternative IPM.

(b) The PSA has also requested that the candidate information pack is expanded to include further information for candidates on their scrutiny role. We will be updating the candidate pack to reflect this recommendation.

2.4. In terms of the recruitment timetable, the key dates and stages in the process have been confirmed with the Privy Council. Initial advertisements will be published in September 2018, with the confirmation of Council appointments expected in March 2019. This timetable includes the required stages of the PSA scrutiny process and the Privy Council approval process.

\section{(b) Confirmation of reappointments}

2.5. Separate to the process for open competition (confirmed in July 2018), Council is asked to agree the process for filling the remaining vacancies through a reappointments process. There are currently three Council members who are eligible for reappointment in April 2019.

2.6. Council should also consider the following when deciding on whether to use reappointments:

- assess and consider the current and future needs of the Council for particular skills and expertise; 
- consider the balance between continuity and refreshment of the Council's membership. The aim should be to produce a degree of change which minimises the risks of stagnation, on the one hand, and instability and delays, on the other;

- consider the diversity of backgrounds within the Council's membership; and

- take account of any relevant external factors, for example, anticipated changes to the constitution of the Council.

2.7. Taking the above factors into account there appears to be no reason why Council cannot proceed with its general principle of using a mixture of open competition and reappointments for 2019 .

- At its meeting in July 2018, Council considered the skills mix of the members, resulting in updates to the selection criteria and competences.

- Given that there will be five vacancies appointed to by open competition, additional reappointments will address the issue of balancing continuity and refreshment of the membership; and

- there are no planned changes to the Council's constitution.

2.8. Should Council approve the use of reappointments for the relevant vacancies, this process would run concurrently with the open recruitment process, which starts in September 2018. It will then be necessary to consult the Privy Council on the planned timing of the reappointment recommendation. In line with PSA guidelines, this should not normally be more than six months before the members' current terms end (October 2018 to March 2019).

2.9. We are not required to submit formal Advance Notice of the reappointments process to the PSA (in the same way as the process for open competition. However, once candidates have been recommended, the scrutiny and recommendation processes are the same as those for open competition.

\section{Communications}

3.1. Subject to Council's decision, we will contact those members who are eligible for reappointment and begin the next steps in the process.

\section{Resource implications}

4.1. The recruitment and selection process, for both open competition and reappointments, will be carried out within existing budgets. 


\section{Equality and diversity}

5.1. Our equality and diversity strategy was set out in detail to the Council previously and incorporated into our recruitment processes going forward.

\section{Risk implications}

6.1. An appropriate and robust process for recruiting and selecting Council members is an essential step in ensuring good governance within the GPhC.

6.2. It is essential that our procedures meet the requirements of the PSA's Section 25c scrutiny process. Failure to ensure that our appointment process meets the four principles of merit, fairness, transparency and openness, and inspiring confidence in regulation means that the PSA may not have confidence in our process. This would result in the Privy Council not making the appointments we recommend.

\section{Monitoring and review}

7.1. Council will be kept updated and informed on the appointments process as this moves forward.

\section{Recommendations}

The council is asked to:

i. Note the progress update on the recruitment of Council members through an open competition process

ii. Agree the process for filling the remaining vacancies through a reappointments process

\section{Laura McClintock, Chief of Staff}

General Pharmaceutical Council

laura.mcclintock@pharmacyregulation.org

Tel 02037138079

5 September 2018 


\section{General \\ Pharmaceutical \\ Council}

Minutes of the Audit and Risk Committee meeting held on Tuesday 17 July

2018 at 25 Canada Square, London at 10:30

TO BE CONFIRMED 23 OCTOBER 2018

Minutes of the public session

Present

Digby Emson (Chair)

Helen Dearden

Mark Hammond

Mohammed Hussain

\section{Apologies}

Jayne Salt

Megan Forbes (Deputy Chief Executive and Director of Operations)

In attendance

Duncan Rudkin (Chief Executive and Registrar)

Claire Bryce-Smith (Director of Insight, Intelligence and Inspection)

Laura McClintock (Chief of Staff)

Tarun Chotai (Interim Head of Finance and Procurement)

Pascal Barras (Risk and Assurance Manager)

Bobbi Birk (Risk and Assurance Analyst)

Sarah Hillary (Moore Stephens)

Michelle Debique (Moore Stephens)

My Phan (Head of Data and Insight) - item 7

Carole Gorman (Governance and Assurance Manager) - item 9

Helen Dalrymple (Council Secretary) 


\section{Attendance and introductory remarks}

14.1. The Chair welcomed those present to the meeting. Apologies had been received from Jayne Salt and Megan Forbes.

\section{Declarations of interest}

15.1. Members were asked to declare any interests at the start of each item.

\section{Minutes of the last meeting}

16.1. The minutes of the public session of the meeting held on the 22 May 2018 were agreed as a true record.

\section{Actions and matters arising}

17.1. Duncan Rudkin (DR) gave an update on action 6.14. from the meeting on 23 May 2017. The action stated that an external review of EDI data in Fitness to Practise procedures would be commissioned. DR said that a law firm had been identified who would help the organisation to do this. Scoping had revealed this area to be complex and a paper would go to Council in September summarising the issues and seeking a steer on next steps. This action was also on the action log for Council and so would be taken off this one.

17.2. Regarding action reference 24.30. from the meeting on the 25 October 2017, Pascal Barras (PB) said that there would be an assurance review of the integrity of the Register at the next meeting in October.

17.3. In reference to action 5.8. from the last meeting on the 22 May, Laura McClintock (LM) outlined the GPhC's current whistle-blowing policy and procedures, including the roles of the whistle-blowing leads, who have special responsibility and training for these types of concerns. Any queries or suggestions for updating it would be welcomed.

17.4. The committee suggested that a 'whistleblowing champion' be nominated who was external to the organisation's processes and could be called upon when it would not be appropriate to use internal systems. This was in line with best practice and it was worth taking further as it was a live issue in the current regulatory climate.

17.5. The committee also suggested that a reference to the grievance policy be added. It was agreed that an updated whistleblowing policy and process would come to a future meeting for approval. 
ACTION: LM

17.6. All other actions were in hand or due to be covered at this meeting.

\section{Update on never events framework and policy}

18.1. Laura McClintock (LM) presented 18.07.ARC.01, this paper updated the committee on the never events framework and policy.

18.2. One member suggested that it would be helpful to add to paragraph 3.2. the thinking behind the fundamental importance of maintaining the Register. Another suggested that the second never event might read better if it was more focused on outcomes in the style of the first never event. It was also asked whether data breaches should be included in the list. LM said that the comments would be fed into the working group's next meeting where updates would be considered.

18.3. In terms of monitoring and review members asked whether there could be a report to Council on the occurrence of never events even if this is a nil return. DR said that the structure was in place for them to receive this through the minutes of this committee, it would also be considered whether it could perhaps be added to the template of Annual Report.

18.4. The committee liked the focus of never events on the integrity of the Register, the key purpose of the organisation. The fact that other potential never events had been considered but rejected on the basis of the criteria demonstrated maturity in keeping these matters simple and avoided creating a 'worry factor'. Perhaps some links to related policies could be included.

18.5. The Committee also discussed how to ensure staff are sighted on never events and what is expected of them. LM told members that there was a communications strategy in place to make sure that staff understood what never events were and their duty to report them. This would fit in with work that was ongoing into improving the culture of the organisation.

18.6. The Committee noted the update on the never events framework and policy and how this would be aligned with the existing incident management reporting and learning (IMRL) procedures.

\section{Internal audit performance report $2018 / 19$ Q1}

19.1. PB presented 18.07.ARC.02. This paper provided a quarterly report to the committee on the progress of the Internal Audit Plan and the follow up of recommendations.

19.2. The committee were pleased to hear that work was progressing according to schedule and that the scheduled audits were on track for timely completion, avoiding the pressures of Q4 that had occurred last year. No audits were scheduled for completion in Q1. 
19.3. The $2018 / 19$ internal audit plan was circulated. PB clarified that the completion dates on the plan had all been agreed by the committee and that they would only be changed with the committee's approval.

19.4. The committee:

i. Noted Q1 2018/19 internal audit plan progress; and

ii. Noted the GPhC's performance in implementing the agreed recommendations.

20. Update from Insight, Intelligence and Inspection

20.1. Claire Bryce-Smith (CBS) presented the update which told the committee about the progress that had been made on data and insight work since January and what the next steps would be.

20.2. The committee welcomed the information and asked to see a critical path for the next 12 months with milestones highlighted. The data and insight work underpinned the development of the organisation's strategy.

ACTION: CBS

20.3. The committee were reassured by DR that this work had a high level of staff engagement, there was a strong appetite for its conclusion as it would aid the crystallisation of strategy. The committee looked forward to a further update in October.

\section{Anti-bribery policy review}

21.1. LM presented 18.07.ARC.05. The paper asked the committee to agree the anti-bribery policy, effective from July 2018.

21.2. The committee suggested putting para 4.2. of the policy, which explained the conditions under which it was acceptable to offer gifts and hospitality, nearer the front of the document as a useful definition.

21.3. Staff engagement with policies and procedures was discussed. LM said that there were various internal channels of communication for these, it could be a good idea to have them as standing items at team meetings; perhaps regularly dealing with different themes.

21.4. The committee agreed the updated anti-bribery policy, effective from July 2018.

\section{Assurance review - General Data Protection Regulation (GDPR)}

22.1. PB presented 18.05.ARC.06, an assurance review of the implementation of GDPR. Carole Gorman (CG) was in attendance. 
22.2. Two challenges that the review had identified were how to maintain the momentum of adhering to the GDPR once the initial attention had died down and the scheduling of the implementation of retention schedules.

22.3. An impact on the pace of GDPR compliance had been that the lists of contracts held by the organisation was incomplete and had to be populated. The committee asked whether this was symptomatic of any other issues.

22.4. Tarun Chotai (TC) explained that some contracts had been held locally by separate teams rather than centrally. Work was underway on a clearer scheme of delegation and a contracts database, these would be ready for a relaunch of the procurement policy in a few months' time.

22.5. The committee agreed that they would like to see an assurance review of the procurement process by their meeting in July 2019.

ACTION: TC

22.6. Para 2.8. of the paper, 'ICO step 7 - Children' was discussed. CG confirmed that children were individuals under the age of 18 years. The committee asked whether this would affect some trainee pharmacy technicians. CG said that she would find out and update the committee in due course.

ACTION: CG

\subsection{The committee noted the paper.}

\section{Any other public business}

23.1. There being no further public business to discuss, the meeting closed at 11:55.

\section{Date of the next meeting:}

Tuesday 23 October 2018 